\title{
A GRHL3-regulated repair pathway suppresses immune-mediated epidermal hyperplasia
}

\author{
William M. Gordon, ${ }^{1,2}$ Michael D. Zeller, ${ }^{1,3}$ Rachel H. Klein, ${ }^{1}$ William R. Swindell, ${ }^{4}$ Hsiang Ho, ${ }^{1}$ Francisco Espetia, ${ }^{1}$ \\ Johann E. Gudjonsson, ${ }^{4}$ Pierre F. Baldi, ${ }^{2,3}$ and Bogi Andersen ${ }^{1,2,5}$ \\ 'Department of Biological Chemistry, School of Medicine, ${ }^{2}$ Center for Complex Biological Systems, and ${ }^{3}$ Department of Computer Science, University of California Irvine (UCI), Irvine, California, USA. \\ ${ }^{4}$ Department of Dermatology, University of Michigan, Ann Arbor, Michigan, USA. ${ }^{5}$ Department of Medicine, School of Medicine, UCI, Irvine, California, USA.
}

\begin{abstract}
Dermal infiltration of T cells is an important step in the onset and progression of immune-mediated skin diseases such as psoriasis; however, it is not known whether epidermal factors play a primary role in the development of these diseases. Here, we determined that the prodifferentiation transcription factor grainyhead-like 3 (GRHL3), which is essential during epidermal development, is dispensable for adult skin homeostasis, but required for barrier repair after adult epidermal injury. Consistent with activation of a GRHL3-regulated repair pathway in psoriasis, we found that CRHL3 is upregulated in lesional skin and binds known epidermal differentiation gene targets. Using an imiquimod-induced model of immune-mediated epidermal hyperplasia, we found that mice lacking CRHL3 have an exacerbated epidermal damage response, greater sensitivity to disease induction, delayed resolution of epidermal lesions, and resistance to anti-IL-22 therapy compared with WT animals. ChIP-Seq and gene expression profiling of murine skin revealed that while GRHL3 regulates differentiation pathways both during development and during repair from immune-mediated damage, it targets distinct sets of genes in the 2 processes. In particular, GRHL3 suppressed a number of alarmin and other proinflammatory genes after immune injury. This study identifies a GRHL3-regulated epidermal barrier repair pathway that suppresses disease initiation and helps resolve existing lesions in immune-mediated epidermal hyperplasia.
\end{abstract}

\section{Introduction}

Skin infiltration of T cells and activation of the IL-23-Th17 axis play key roles in the initiation and progression of psoriasis $(1,2)$. IL-17 and IL-22 cytokines produced by Th17/Th22 cells stimulate proliferation and inhibit differentiation of epidermal keratinocytes, thus accounting for the marked epidermal hyperplasia characteristic of psoriasis. Secondarily, the epidermis itself produces a range of cytokines and chemokines that help maintain the disease (3-11). While copy number polymorphisms in the $\beta$-defensins and deletions of the $L C E 3 C / B$ locus have been associated with psoriasis (12), a primary role for epidermal defects in the pathogenesis of psoriasis is controversial. In particular, it is unknown whether epidermal repair pathways play important roles in the disease and whether such pathways interact with infiltrating immune cells.

The evolutionarily conserved grainyhead-like 3 (GRHL3; also known as GET1), a member of the CP2-like transcription factor family, is essential for epidermal differentiation and barrier formation at the end of mouse embryogenesis (13-19). Grhl3 deletion leads to decreased expression of a number of genes critical for barrier formation, including those encoding lipid processing enzymes, cell-cell adhesion molecules, and structural proteins (16). In humans, dominant-negative GRHL3 mutations are associated with defective periderm development in Van der Woude syndrome (20). In contrast to its critical role in initial epidermal formation, Grhl3 appears to be dispensable for epidermal differ-

Conflict of interest: The authors have declared that no conflict of interest exists Submitted: May 20, 2014; Accepted: September 18, 2014.

Reference information: / Clin Invest. 2014;124(12):5205-5218. doi:10.1172/JCI77138. entiation during adult skin homeostasis (14). However, a possible role for GRHL3 in adult skin is suggested by the observation that it is one of only 8 genes associated with both altered disease expression and a psoriasis susceptibility locus (21).

We now demonstrate a requirement for Grhl3 in the repair of barrier-disrupting epidermal lesions in adult skin. GRHL3 was upregulated in human psoriasis lesions, where its expression correlated with psoriasis-associated cytokine activity. Furthermore, in imiquimod-treated (IMQ-treated) mice, a frequently used model of psoriasis (22-24), loss of Grhl3 facilitated formation of lesions, delayed their resolution, and conferred treatment resistance, which suggests that a GRHL3-regulated repair pathway suppresses disease activity. Thus, despite being dispensable during normal epidermal homeostasis, Grhl3 was again required for barrier repair after immune-mediated epidermal damage in adult skin. While this activity was reminiscent of its critical role in embryonic epidermal differentiation, GRHL3 targeted a distinct set of genes in adult epidermal repair.

\section{Results}

GRHL3 is dispensable for homeostatic adult epidermal barrier formation, but required for injury repair in the adult. To test whether Grhl3 plays a role in adult epidermal repair, we crossed mice (C57BL/6) in which the exons of the DNA-binding domains of Grhl3 had been floxed (18) with mice expressing Cre under the control of the Krt14 promoter (Supplemental Figure 1A; supplemental material available online with this article; doi:10.1172/JCI77138DS1; and ref. 25). The resulting pups with conditional KO of Grhl3 in epi- 
A
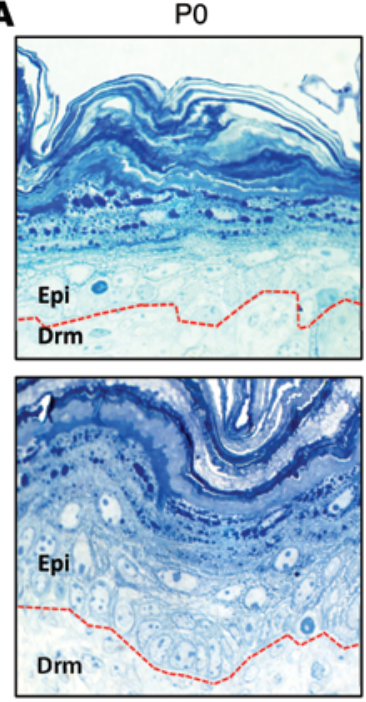

B

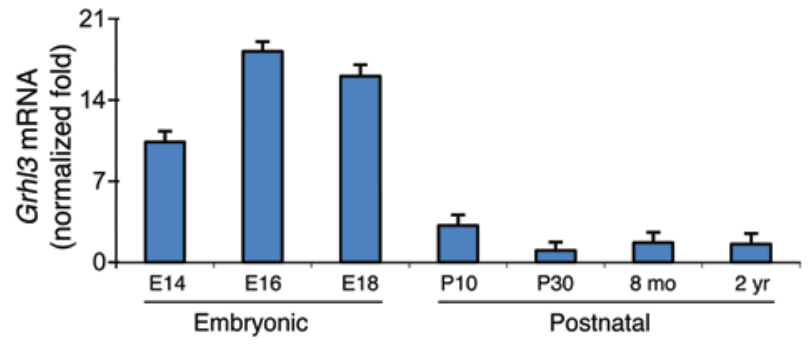

D

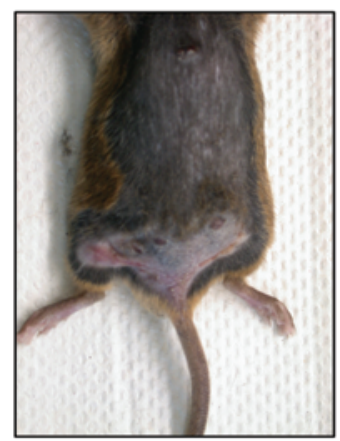

Injured cKO + 3 mo
P5

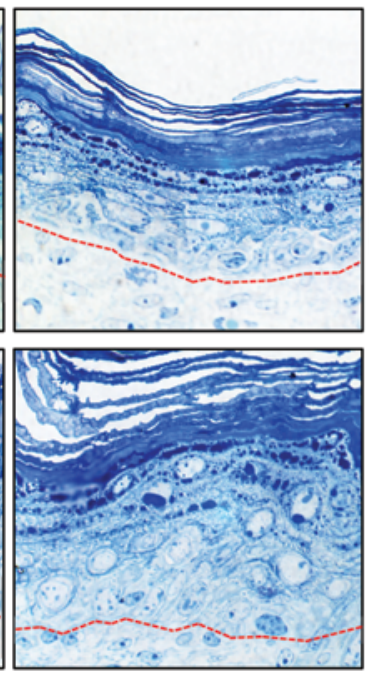

P10
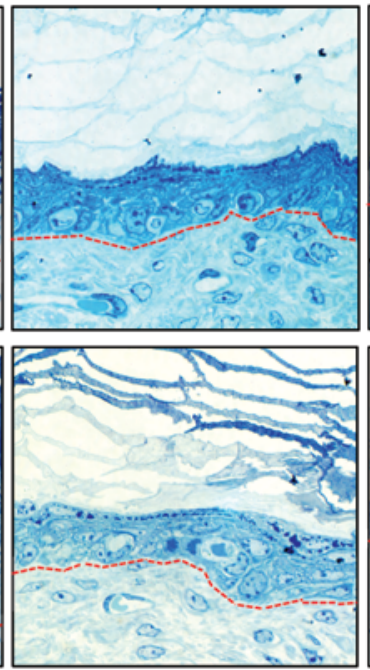
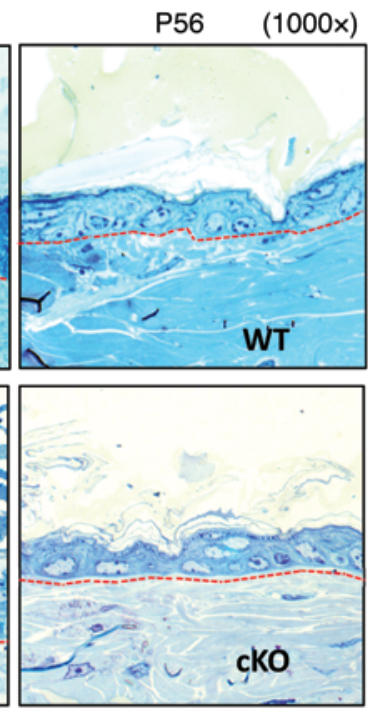

C
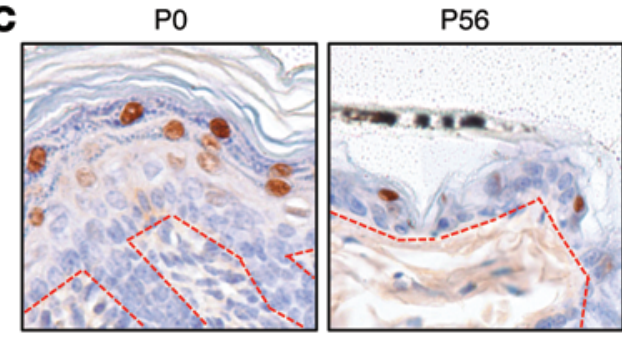

$\beta$-Gal $(400 x)$

E
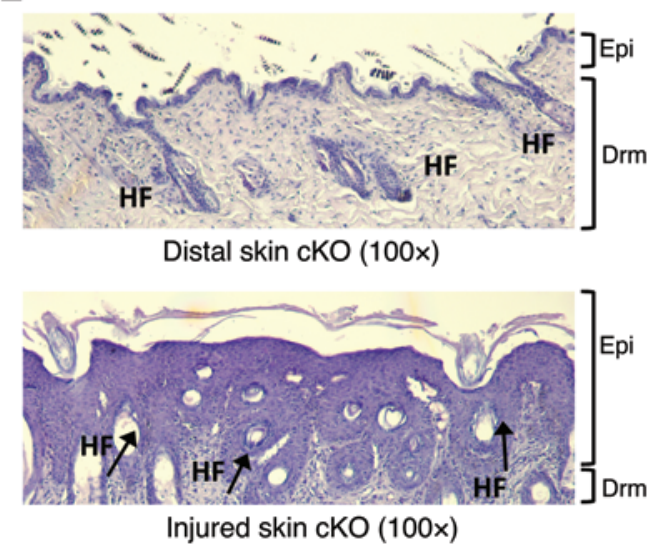

$\mathbf{F}$

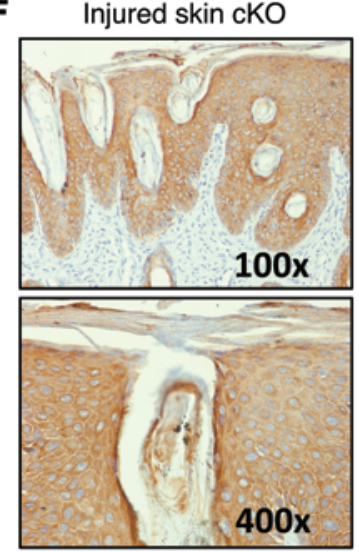

KRT6 IHC

Figure 1. Grhl3 is dispensable for epidermal differentiation in the adult mouse, but is required for resolution of epidermal injury. (A) Toluidine blue staining of WT $(n=2)$ and Grh/3 cKO (Krt14-Cre Grh/3/f/f; $n=2)$ skin at the indicated postnatal days. Dashed red line denotes basal lamina separating epidermis (Epi) from dermis (Drm). Original magnification, x1,000. (B) Grhl3 mRNA expression, by quantitative PCR, in WT skin during embryonic development and postnatally ( $n=3$ mice per time point). (C) $\beta$-Gal IHC in Grh/3-Cre/LacZ reporter mice at PO and P56. Dashed red line denotes basal lamina. Original magnification, $\times 400$. (D) Nonhealing abrasive injury (received 3 months earlier) in a Grh/3 cKO mouse. (E) H\&E staining of unaffected and affected regions of Grhl3 cKO skin 3 months after injury. Hair follicles (HF) are indicated. Original magnification, $\times 100$. (F) KRT6 IHC in the affected area of a Grhl3 cKO nonhealing abrasive injury. Original magnification, $\times 100$ (top); $\times 400$ (bottom).

thelium (Krt14-Cre Grhl3 ${ }^{\text {flff; }}$; referred to herein as Grhl3 cKO mice) were born in proper Mendelian ratios (data not shown). However, in contrast to the Grhl3 germline KO (Grhl3 KO), Grhl3 cKO mice showed no spina bifida, exencephaly, or eye-open features at birth and had a milder epidermal phenotype (Supplemental Figure 1B). Histological and transmission electron microscopy (TEM) analy- ses of Grhl3 cKO epidermis at birth showed mild hyperplasia and impaired terminal differentiation, with rounder cell morphology beneath the stratum corneum (Figure 1A and Supplemental Figure 1C). This phenotype persisted through P5, was resolving by P10, and had normalized by P56, both by gross appearance and by histology (Figure 1A and Supplemental Figure 1D). In addition, adult 
A

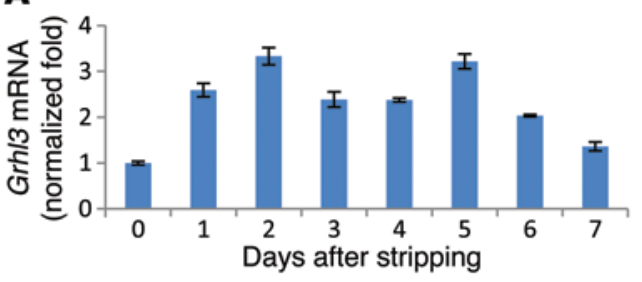

C

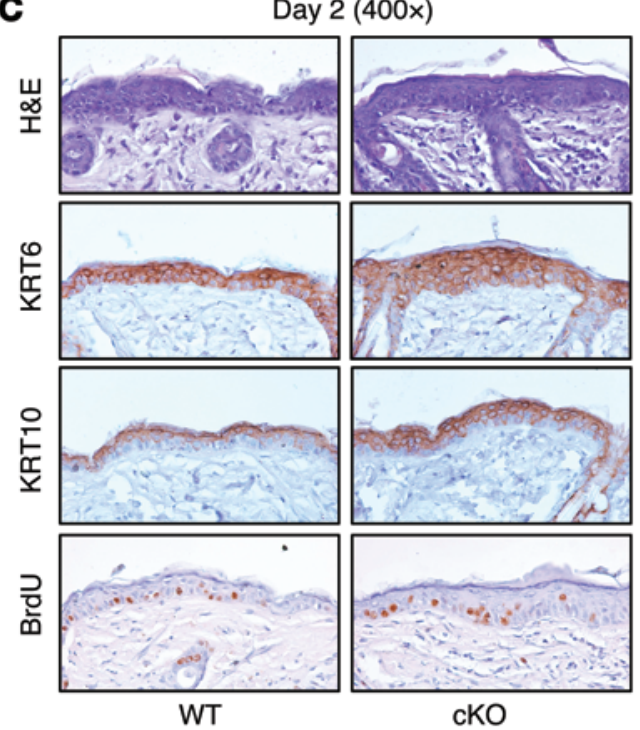

F

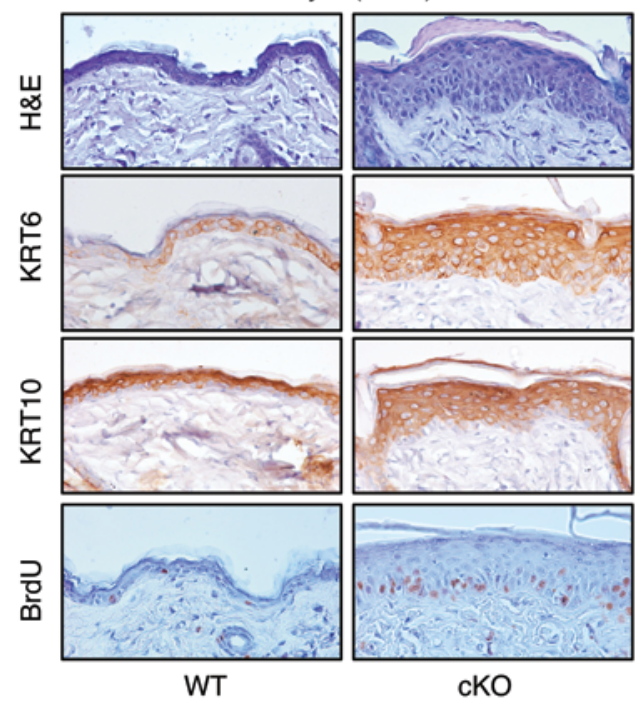

B

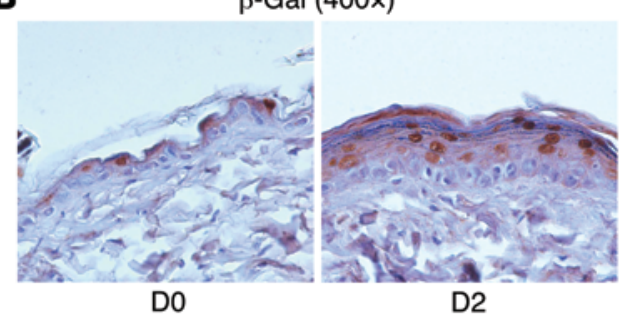

D

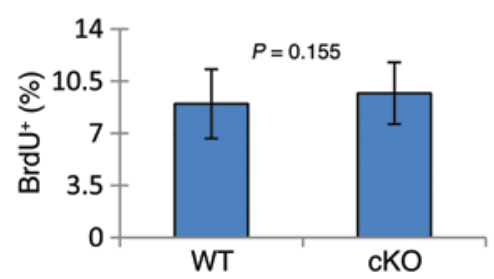

E

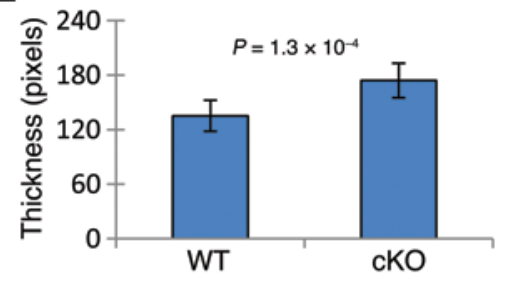

G

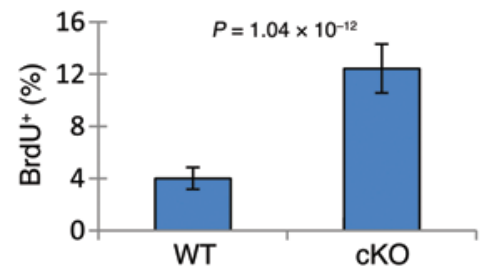

H

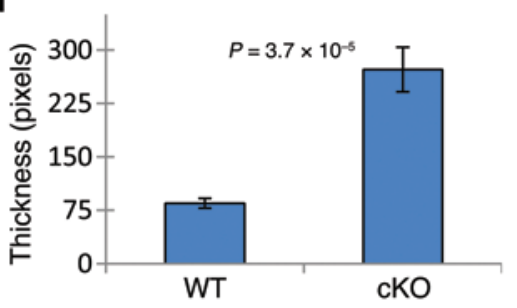

Figure 2. Loss of Grhl3 impairs adult mouse epidermal barrier repair. (A) Grh/3 mRNA expression (quantitative $P C R$ ) in WT skin after wax stripping. (B) $\beta$-Gal IHC in Grhl3-Cre/LacZ reporter mice before (DO) and 48 hours after (D2) wax stripping $(n=2)$. (C-H) Grhl3 cKO skin 48 hours (C-E) and 4 days $(\mathbf{F}-\mathbf{H})$ after wax stripping $(n=3$ per group). (C and $\mathbf{F})$ Representative $\mathrm{H} \& \mathrm{E}$ and KRT6, KRT10, and BrdU IHC. Original magnification, $\times 400$. (D and $\mathbf{C})$ Quantification of BrdU staining (mean and SEM; $P$ values indicated). ( $\mathbf{E}$ and $\mathbf{H}$ ) Quantification of epidermal thickness (mean and SEM; $P$ values indicated). (I) TEWL in skin after wax stripping from day 5 through day 9 (mean and range; $n=5$ [WT], 4 [Grhl3 cKO]). ${ }^{*} P<0.05$.

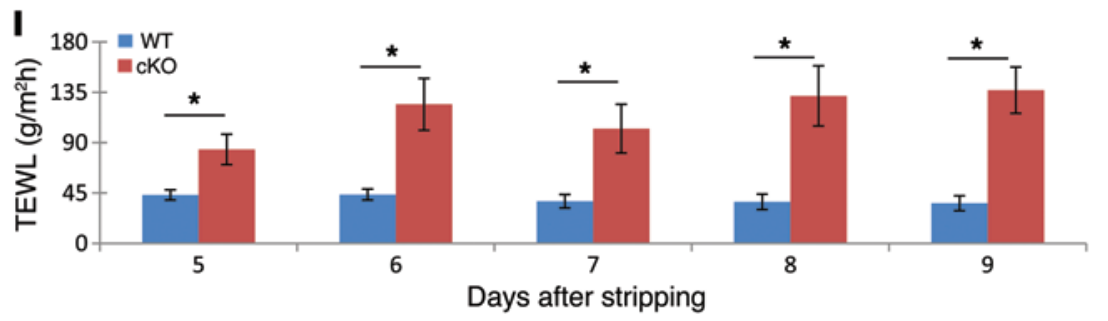


Grhl3 cKO skin showed no significant increase in a key measurement of epidermal barrier function, trans-epidermal water loss (TEWL) (Supplemental Figure 1E). Despite the normal adult Grhl3 cKO skin phenotype, we observed mild gene expression changes similar to those found in the germline Grhl3 KO skin at birth (Supplemental Figure 1F), suggesting the possibility of subclinical epidermal defects. The phenotypic improvement over time (Figure 1A) correlated with the normal pattern of Grhl3 mRNA levels in WT mice (Figure 1B), which peaked during embryogenesis, followed by decreasing expression through P10. Consistently, in Grhl3-Cre/LacZ reporter mice (26), a dense layer of GRHL3positive cells was observed at the top of the suprabasal compartment at birth, whereas the adult epidermis contained only widely spaced GRHL3-positive cells (Figure 1C). These data showed that whereas Grhl3 is essential during earlier stages of epidermal formation, it becomes progressively less important over the course of development, and is dispensable for epidermal barrier formation during adult epidermal homeostasis.

During observation of adult Grhl3 cKO animals, several mice developed abrasive skin lesions after fighting. When these mice were separated and allowed to heal, the affected skin areas did not return to normal, even after 3 months (Figure 1D). Histological skin examination showed a highly hyperplastic epidermis, whereas unwounded adjacent skin was normal (Figure 1E). The hyperplastic epidermis was positive for KRT6, a marker of aberrant keratinocyte differentiation and injury response, and the stratum corneum was flattened and highly compacted (Figure 1F), reminiscent of the neonatal Grhl3 KO epidermis (16). These observations suggest that Grhl3 is required for skin healing after abrasive skin injury.

GRHL3 is required for epidermal barrier repair and suppression of epidermal hyperplasia after mechanical and chemical barrier disruptions. To systematically investigate the role of Grhl3 in adult epidermal repair, we used barrier disruption via wax stripping, physically removing the upper suprabasal layers of the dorsal backskin epidermis in Grhl3 cKO animals. Grhl3 mRNA expression increased 3 -fold by 48 hours after wax stripping, staying elevated for several days (Figure 2A). An increased number of GRHL3-expressing cells was also visualized at 48 hours in the Grhl3-Cre/LacZ reporter mice (Figure 2B). In WT mice, epidermal barrier dysfunction (TEWL) occurred immediately after wax stripping, worsening over the next 24-72 hours before returning to normal by day 7 (Supplemental Figure 2A). Similarly, basal cell proliferation, epidermal thickness, and KRT6 expression increased dramatically during the first $24-48$ hours, returning to baseline by day 7 (Supplemental Figure 2, B-D). Therefore, wax stripping represented a reproducible injury model suitable for studying the dynamic role of Grhl3 in epidermal barrier repair.

A mild but clear phenotype was observed within 2 days of recovery after wax stripping in Grhl3 cKO mice. While there was no difference in basal cell proliferation between the 2 genotypes at this stage (Figure 2D), the Grhl3 cKO epidermal thickness was already significantly increased (Figure 2, C and E). This suprabasal hyperplasia (Figure 2C) was similar to that found in the neonatal epidermis of Grhl3 KO mice (16). By day 4 after wax stripping, the WT epidermis showed clear signs of a repair response, with decreased keratinocyte proliferation and a thinner epidermis. In contrast, the Grhl3 cKO epidermis failed to resolve the barrier damage, exhibiting persistent keratinocyte proliferation and increased thickness of the suprabasal epidermis, which was marked by KRT10 and KRT6 expression (Figure 2, F-H). As evaluated by TEWL, damage from stripping was exacerbated in Grhl3 cKO mice, which showed a clear failure to repair the barrier defect (Figure 2I). When Grhl3 cKO wax-stripped mice were allowed to recover for 2 or 3 full weeks, they continued to show significant hyperplasia of their epidermis, with a strong presence of $\mathrm{CD} 45^{+}$ immune cells within the dermis and along the epidermal-dermal junction (Supplemental Figure 3). Similar results to stripping were found after topical skin application of SDS, which disrupts the epidermal barrier by removing the protective epidermal lipids (27); after 2 days of recovery, Grhl3 cKO mice showed more severe damage, hyperplasia, and increased TEWL (Supplemental Figure 4, A and B). Together, these observations demonstrated that Grhl3 is critical for the suppression of epidermal hyperproliferation and reconstruction of the epidermal barrier during repair of a barrierdisrupting injury in adult skin.

Activation of a GRHL3-regulated epidermal repair pathway in human psoriasis lesions. Given GRHL3's critical role in adult epidermal barrier repair, we evaluated its expression in published gene expression databases from human psoriasis $(28,29)$; the disease has been associated with a disrupted epidermal barrier (30). While GRHL3 was similarly expressed between normal and uninvolved psoriasis skin, it was found to be consistently and significantly upregulated 2.62-fold in psoriasis lesions in 3 independent datasets (Figure 3, A and B, and refs. 31-33). Intriguingly, patients with stronger upregulation of GRHL3 expression (lesional versus uninvolved) also showed stronger upregulation of genes induced by IL-17 and IL-22 $\left(P \leq 1 \times 10^{-10}\right.$ and $P \leq 3.03 \times 10^{-5}$, respectively; Figure 3C, Supplemental Table 1, and ref. 34). These data suggested that GRHL3 expression correlates with the intensity of key immune drivers of this disease, which led us to hypothesize that its upregulation represents activation of an epidermal repair pathway in psoriasis lesions in response to barrier disruption.

To begin investigating the above hypothesis, we compared our prior GRHL3 siRNA knockdown gene expression and GRHL3 ChIP-Seq datasets from normal differentiating human keratinocytes (NHEK) (35) with the human psoriasis gene expression data (31-33). Of the 1,206 GRHL3-regulated genes in differentiating NHEKs, 312 were also differentially expressed in human psoriasis lesions compared with nonlesional skin (overlap significant, $P \leq 3.65 \times 10^{-5}$; Figure 3D and Supplemental Figure 5A). Of these 312 genes, 47 contained proximal GRHL3 ChIP peaks ( $+10 \mathrm{~kb}$ to -5 $\mathrm{kb}$ of transcription start site [TSS]; overlap significant, $P \leq 0.0485$; Figure $3 \mathrm{E}$ and Supplemental Figure $5 \mathrm{~B}$ ). The psoriasis expression of 198 of the overlapping genes was altered in the direction predicted by the GRHL3 knockdown experiment (Figure 3D and ref. 35), supporting the existence of a GRHL3-regulated gene expression pathway in lesional psoriasis. The differentially regulated genes that were altered in the predicted direction (Figure 3G) showed enrichment for epidermal cell differentiation, response to wounding, immune response, and lipid transport (Supplemental Figure 5C); the 82 likely direct targets (Figure 3E) included the known epidermal genes CNFN, ELF3, ELOVL7, IVL, and OVOL1 as well as IGFL2, which has previously been shown to be repressed in psoriatic skin (Figure 3G and ref. 36). GRHL3 bound directly to 

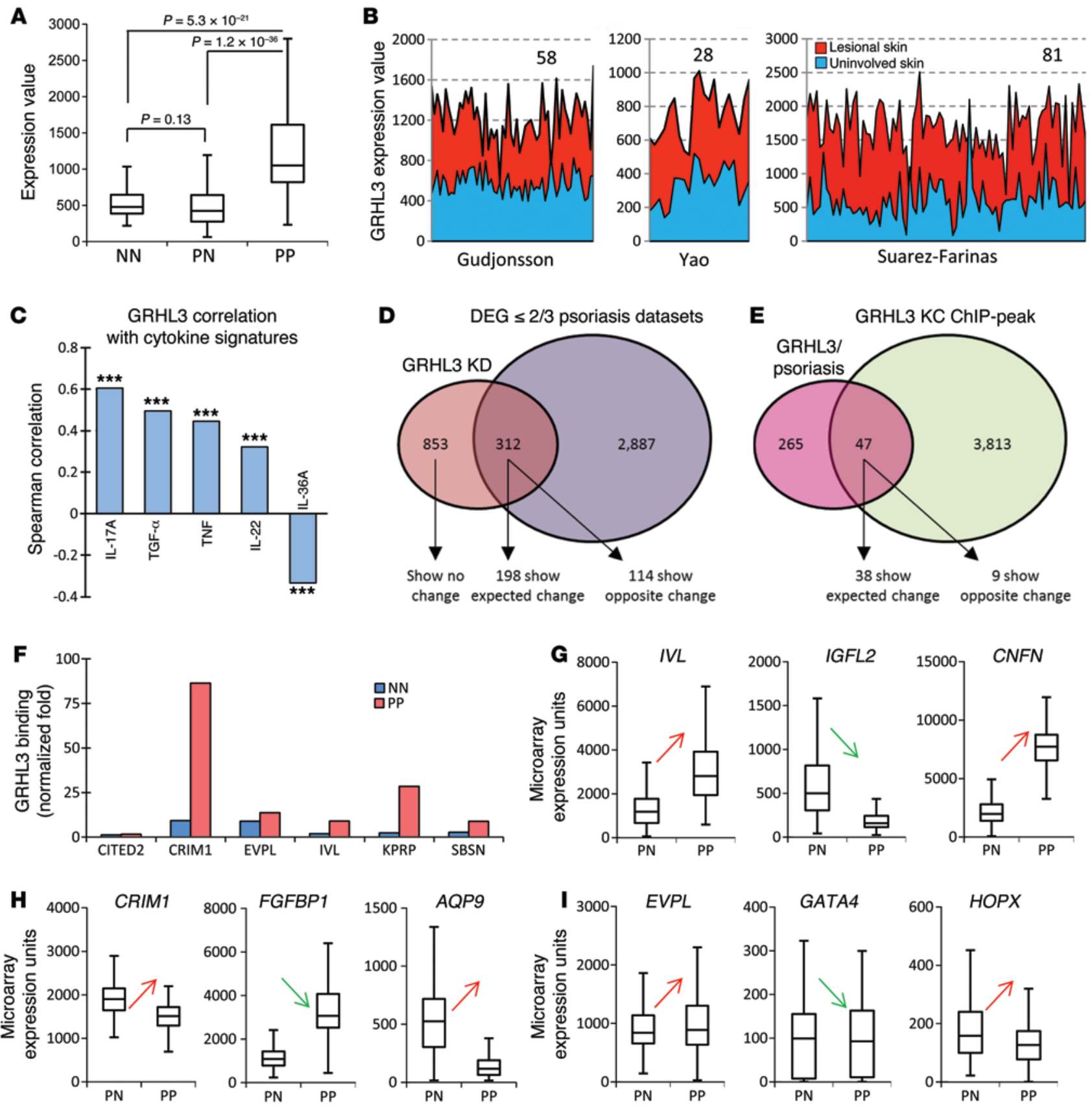

Figure 3. A GRHL3-regulated epidermal repair pathway in human psoriasis. (A) Relative GRHL3 mRNA expression in normal (NN), uninvolved (PN), and psoriatic lesions (PP) from published datasets (31-33). (B) GRHL3 expression in individuals from 3 independent experiments (refs. 31-33; $N$ for each dataset indicated within graph). (C) Top Spearman correlations of GRHL3 expression to cytokine gene signatures (75) in lesional psoriasis. ${ }^{* * *} P<0.001$. (D) Overlap of DEGs in GRHL3 knockdown (KD) human keratinocytes with DEGs in human psoriatic lesions versus uninvolved skin. (E) Overlap of DEGs in common in $\mathbf{D}$ and the GRHL3 human keratinocyte (KC) ChIP-Seq peaks ( +10 to $-5 \mathrm{~kb}$ of TSS). (F) GRHL3 binding (ChIP-PCR) to the indicated gene targets in normal and lesional psoriasis skin. (G-I) Examples of GRHL3-regulated genes in psoriasis lesional skin that, based on the GRHL3 RNAi experiments in human keratinocytes, were regulated in the expected manner $(\mathbf{G})$, opposite the expected manner $(\mathbf{H})$, or unchanged (I) compared with normal skin. Red arrows, predicted upregulation; green arrows, predicted downregulation. (A and $\mathbf{G}-\mathbf{I})$ Boxes denote IQR, lines denote median, upper whiskers denote lesser of either (quartile $3+1.5 \times I Q R$ ) or maximum value, lower whiskers denote greater of either (quartile $1-1.5 \times I Q R$ ) or minimum value.

a subset of putative GRHL3 target genes in normal human epidermis, including the prodifferentiation gene $I V L$, and this binding was enhanced in lesional epidermis (Figure 3F).

The subset of overlapping genes (Figure 3, D and E) whose psoriasis expression did not go in the direction predicted by the GRHL3 knockdown experiments showed enrichment in cell cycle, proliferation, and epidermal morphogenesis (Supplemental Figure 5D). These genes included potentially interesting GRHL3 targets such as CRIM1, which is involved in adhesion and migration (37), FGFBP1, whose upregulation is associated with epidermal cancer $(38,39), A Q P 9$, which encodes a granular protein that may function in the epidermal barrier $(40,41)$, and the suprabasally 
A

B

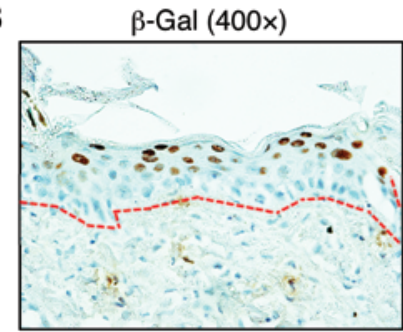

$5 \% \mathrm{IMQ}$ day 3
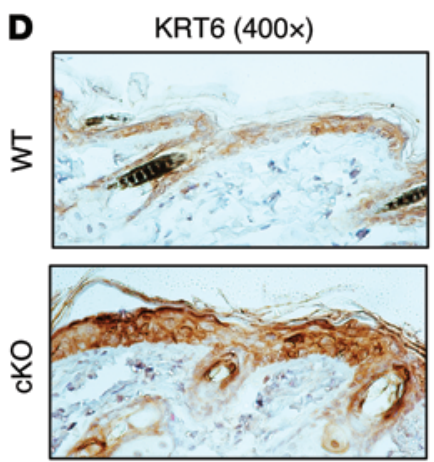

6 days of $0.25 \% \mathrm{IMQ}$ treatment
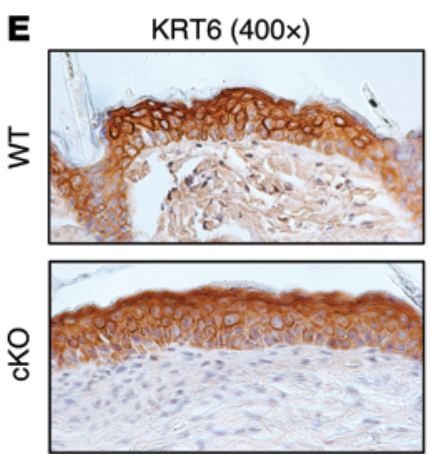

6 days of $1 \%$ IMQ treatment

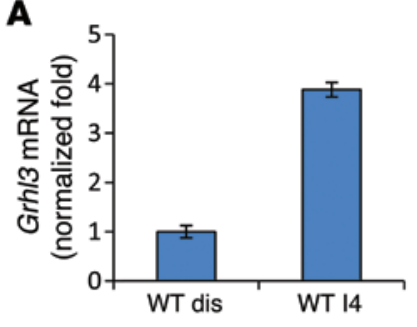

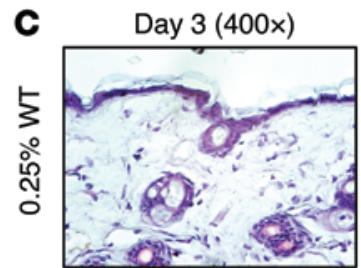
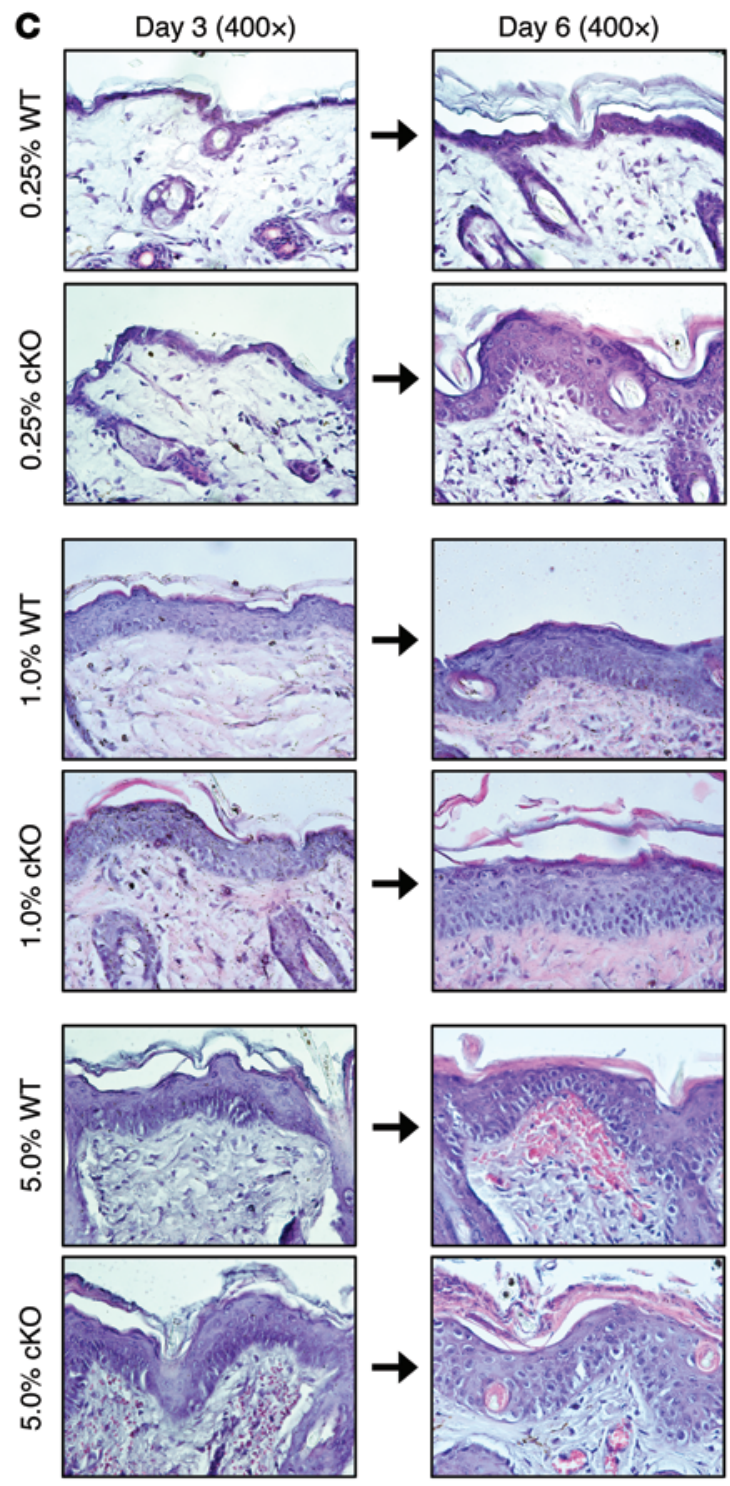

F
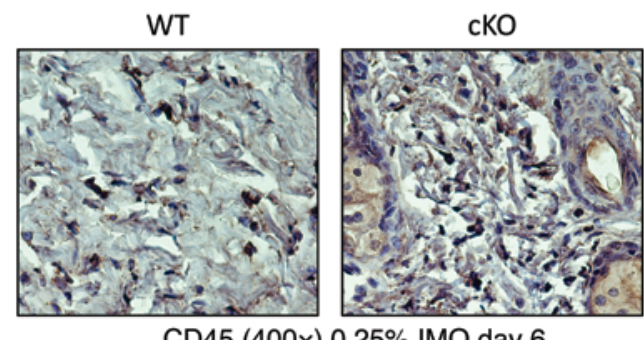

CD 45 (400x) $0.25 \%$ IMQ day 6
Figure 4. Loss of Grhl3 increases susceptibility to immune mediated epidermal pathology. (A) Grhl3 mRNA expression in epidermis after 4 days of IMQ treatment (WT 14) compared with distal untreated skin (WT dis) $(n=3)$. (B) $\beta$-Cal IHC in Grh/3-Cre/LacZ reporter mice after 3 days of IMQ treatment. Dashed red line denotes basal lamina. Original magnification, $\times 400$. (C) H\&E of WT and Grhl3 cKO skin at days 3 and 6 of treatment with $0.25 \%, 1 \%$, and $5 \%$ IMQ. Original magnification, $\times 400$. (D) KRT6 IHC of WT and Grhl3 cKO skin at day 6 of $0.25 \%$ IMQ treatment. Original magnification, $\times 400$. (E) KRT6 IHC of WT and Grhl3 cKO skin at day 6 of $1 \%$ IMQ treatment. Original magnification, $\times 400$. (F) CD45 IHC of dermal immune cell infiltration after 6 days of $0.25 \%$ IMQ treatment. Original magnification, $\times 400$.

In light of these data, we also investigated whether GRHL3 expression correlates with psoriatic lesion healing. We identified 4 independent gene expression datasets from clinical trials in psoriasis using anti-TNF (etanercept; 2 independent datasets), anti-IL-23 (guselkumab), or anti-IL-17R (brodalumab) (48-51). Consistent with the gene expression results in Figure 3, in these clinical trials, GRHL3 was significantly upregulated in lesional versus nonlesional skin, and after treatment, this high level was significantly reduced to levels similar to the nonlesional controls in all but 1 etanercept trial, which still showed the correct trend (Supplemental Figure 6, A-D). These data suggest that as immune-based therapies resolve psoriasis lesions, GRHL3 is downregulated, perhaps in response to GRHL3-mediated repair of the epidermal barrier.

Collectively, these data point to the activation of a GRHL3-regulated repair pathway in psoriasis lesions and its potential importance in their resolution. However, a subset of GRHL3 differentiation-related gene targets did not cor-

expressed $D L X 3$, whose repression has been associated with epidermal hyperplasia (42) (Figure 3H). There were also numerous siGRHL3-regulated genes with no expression change in psoriasis; these showed enrichment in epidermal cell differentiation, chromatin assembly, keratinization, response to wounding, and proliferation (Supplemental Figure 5E). These genes included putative GRHL3 targets such as the known epidermal genes KLF4 (a recently identified psoriasis susceptibility gene; ref. 43), EVPL, OVOL2, PPL, SBSN, and HOPX (44) as well as GATA4 $(45,46)$, a regulator of tight junctions (47) (Figure 3I). relate with GRHL3 expression in psoriasis lesions. Interestingly, GRHL3 still bound to some of these misregulated targets, and this binding was enhanced in psoriasis lesions (Figure 3F), which suggests that the psoriasis cellular environment can affect GRHL3's ability to activate or repress genes.

Grhl3 provides resistance to IMQ-induced skin lesions and is required for their repair. To test the function of the putative GRHL3regulated epidermal repair pathway in immune-mediated epidermal injury, we used IMQ, a Toll-like receptor agonist that consistently induces psoriatic-like lesions in mice $(22,52)$ and 
susceptible patients (53-55). IMQ treatment increased epidermal Grhl3 mRNA expression (Figure 4A) and $\beta$-gal staining in Grhl3$\mathrm{Cre} / \mathrm{LacZ}$ reporter mice (compare Figure $4 \mathrm{~B}$ with Figure 1C, right). We first assessed whether loss of Grhl3 alters the dose response of the epidermis to IMQ. Application of 0.25\% IMQ showed no visible damage to WT or Grhl3 cKO skin at day 3, but a striking difference by day 6 . The WT mice showed a very mild histologic response to this low dose, with patchy upregulation of KRT6 and no obvious hyperplasia, whereas Grhl3 cKO mice showed both marked hyperplasia and strong upregulation of KRT6 (Figure 4, C and D). Similarly, after 3 days of treatment with $1 \%$ IMQ, there was no visible difference between WT and Grhl3 cKO mice. However, there was a slightly more prominent phenotype histologically in $\mathrm{Grhl} 3 \mathrm{cKO}$ mice by day 6, with similar upregulation of KRT6 (Figure 4, C and E). When the standard dose of IMQ (5\%) was applied, there was similar visible damage, barrier disruption severity (TEWL), and microscopic injury (H\&E) in WT and Grhl3 cKO mice (Figure 4C and Supplemental Figure 7, A and B). Additionally, enhanced CD45 infiltration was observed in Grhl3 cKO skin at day 6 with $0.25 \%$ IMQ treatment (Figure 4F and Supplemental Figure 7C).

We next assessed whether Grhl3 is required for resolution of epidermal hyperplasia after standard-dose 5\% IMQ treatment. At 4 days after stopping IMQ application, there was marked recovery in the WT mice, but continued hyperplasia in Grhl3 cKO mice; at 12 days of recovery, there were no longer visual signs of epidermal damage in WT mice, in contrast to the continued epidermal hyperplasia and strong KRT6 staining in Grhl3 cKO mice (Figure 5, $A$ and B). Equivalent levels of CD45 infiltration were observed in WT and Grhl3 cKO skin during early recovery from 5\% IMQ (Figure $5 \mathrm{C}$ and Supplemental Figure 7C). To further test the role of Grhl3 in immune-mediated injury, we used anti-IL-22 treatment prior to IMQ application in order to block the immune response. Whereas WT mice responded to anti-IL-22, showing a reduced damage response and nearly normal epidermal thickness, Grhl3 cKO mice showed minimal improvement (Figure 5D). From these observations, we conclude (a) that sensitivity to low-dose IMQ treatment is enhanced in Grhl3-deleted mice with increased epidermal hyperplasia and immune cell infiltration; (b) that Grhl3 is required for the repair of IMQ-induced epidermal pathology; and (c) that loss of Grhl3 confers resistance to anti-IL-22 therapy. Together, these data provide evidence for a GRHL3-regulated epidermal repair pathway that suppresses disease severity in immune-mediated skin disease.

GRHL3 binding is dynamic and condition dependent in epidermal differentiation and barrier formation after epidermal damage. To understand how GRHL3 regulates barrier formation and repair, we used ChIP-Seq to define genome-wide GRHL3 binding sites under 3 different conditions: (a) embryonic epidermal differentiation (i.e., at E16.5), (b) 4 days after wax stripping, and (c) after 4 days of $5 \%$ IMQ treatment. The GRHL3 chromatin binding sites were then linked to global gene expression changes in Grhl3-deleted mice, as determined by microarray analysis, under the 3 different conditions. The GRHL3 antibody used for these ChIP-Seq experiments was previously validated and used in human keratinocytes (35) and was further validated here in a series of mouse skin experiments taking advantage of $\mathrm{Grhl} 3 \mathrm{cKO}$ skin as a negative control (Supplemental Figure 8, A-D).
We identified 4,035 (common to 2 independent biological experiments), 4,820 (1 biological sample) and 9,294 (1 biological sample) significant GRHL3 peaks (FDR < 0.05), respectively, in the E16.5 skin, wax stripping, and IMQ treatment conditions (Supplemental Figure 8E). The peaks identified in each condition showed strong sequence conservation, as measured by Phastcons (Supplemental Figure 8F).

The overlap in GRHL3 peak locations under the 3 different conditions was statistically evaluated using a hypergeometric distribution (56). As expected, we found a substantive and significant overlap between the 2 independent E16.5 ChIP-Seq experiments (42\%, $P<1 \times 10^{-10}$; Figure 6A and Supplemental Figure $\left.8 \mathrm{E}\right)$. In contrast, while still greater than expected by random chance, only $1,391\left(28 \%, P<1 \times 10^{-10}\right)$ of the wax stripping peaks and 1,326 $\left(17 \%, P<1 \times 10^{-10}\right)$ of the IMQ peaks were in common with the E16.5 peaks overlapping between the 2 independent replicates (Figure 6, A and B, and Supplemental Figure 8G). A similar number of overlapping peaks was found when we compared the wax stripping and IMQ peaks with those from individual E16.5 ChIPSeq experiments. Therefore, for subsequent analysis, we used the overlapping E16.5 GRHL3 peaks, the highest-confidence binding events. However, there was also strong sequence conservation over peaks that were only called in either of the E16.5 ChIP-Seq experiments (Supplemental Figure 8F), which indicates that these also contain bone fide GRHL3 binding sites.

Comparing the localization of peaks in the different conditions, we found that the overlap of the 2 E16.5 ChIP-Seqs had the highest percentage of peaks (89\%) located within proximal promoters ( +2 to $-2 \mathrm{~kb}$ around TSS) and gene bodies, while the corresponding numbers for the wax stripping and IMQ experiments were $81 \%$ and $70 \%$, respectively (Figure $6, \mathrm{C}-\mathrm{E}$ ). Compared with the other conditions, IMQ GRHL3 peaks were more commonly found in intergenic regions (28\%) and less commonly found in proximal promoters (19\%) (Figure 6E). These results indicate that chromatin binding of GRHL3 is dynamic, with location highly dependent on the functional state of the epidermis.

GRHL3 binds to multiple related motifs that tend to overlap with known genomic regulatory features. Binding site analysis of ChIPSeq peaks identified previously described GRHL1/3 in vitro binding sites with the GRHL3 position weight matrix (PWM) found in a significant subset of the peaks in both embryonic and wax stripping experiments $(P \leq 0.05$; Figure 6F). Additionally, the TAGteam motifs (CAGGTAG and CAGGAG) recently reported as grainyhead binding motifs in Drosophila (57) were identified in a significant number of peaks in both embryonic and wax stripping experiments $(P \leq 0.05$; Figure $6 \mathrm{~F})$. Unexpectedly, the perfect consensus GRHL3 binding site $(18,58)$ was found in a relatively small fraction of the peaks, and many computationally identified (59) GRHL3 sites across the genome were not bound by GRHL3 (Supplemental Figure 8G). De novo motif calling identified a number of statistically significant motifs in all 3 experiments (overlap of the E16.5 ChIPs, wax stripping, and IMQ), including E2f3, Sp100, Zfx, and Foxa1 in the embryonic peaks; Cebpa, Irf, and Nkx2-1 in the wax stripping peaks; and Nkx3-1, Smad4, and cJun in the IMQ peaks (Supplemental Table 2). Especially notable enrichment of non-GRHL3 binding sites was observed in the IMQ ChIP-Seq peaks. 
A
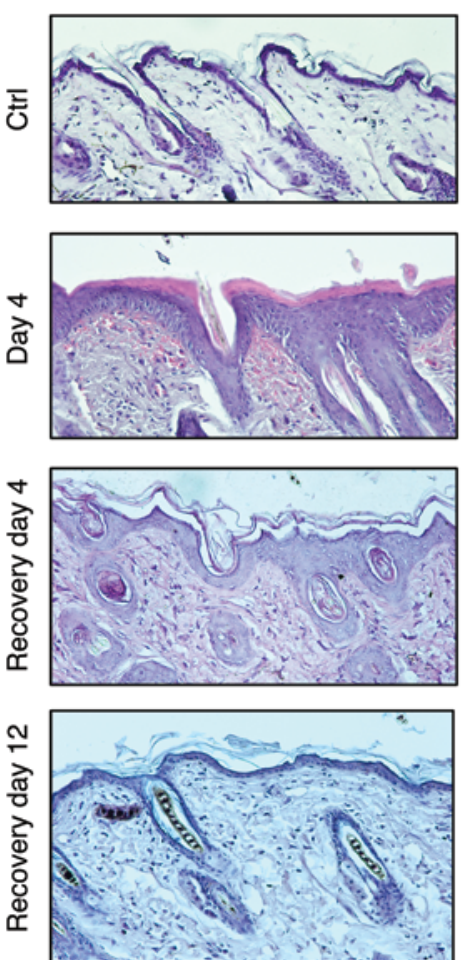

$5 \%$ IMQ treatment $(200 x)$
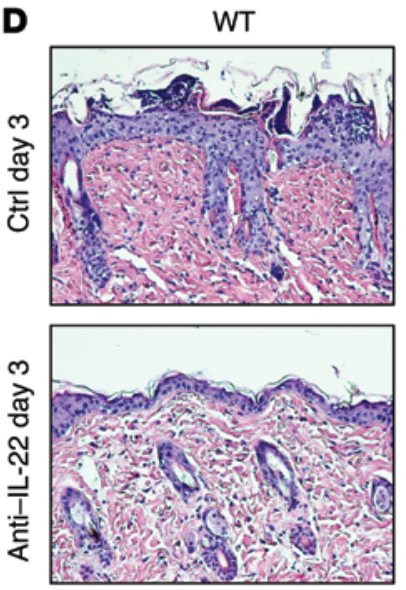

$5 \%$ IMQ treatment $(200 x)$
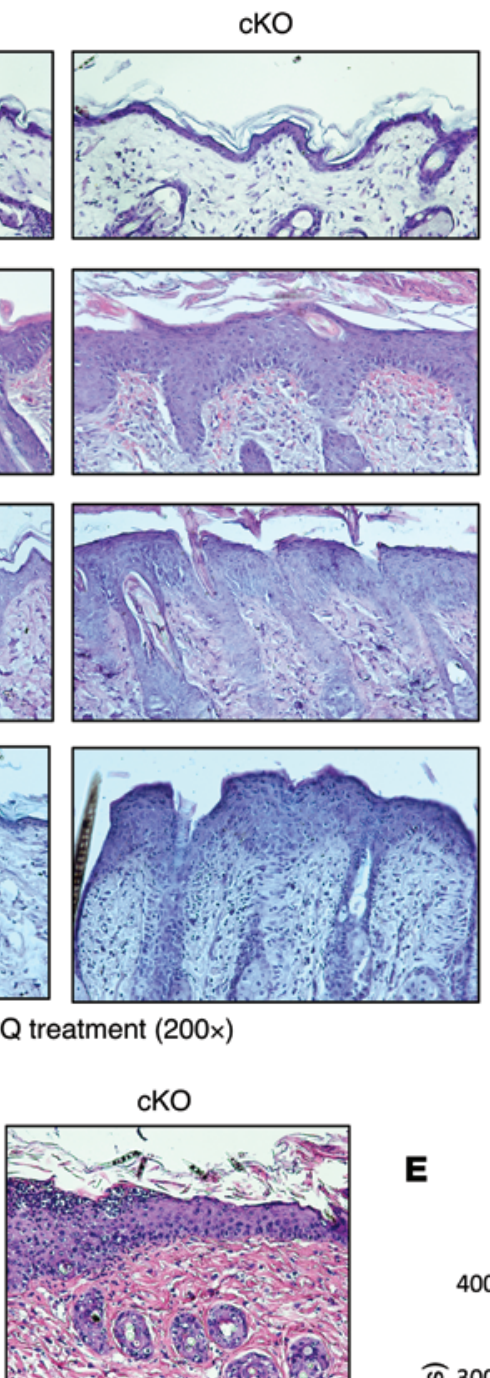

H. hentise

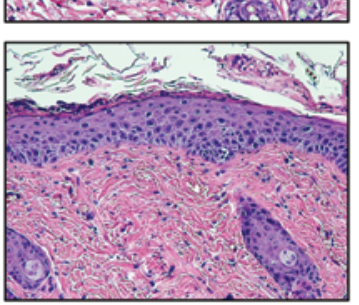

$\mathbf{E}$
In order to better understand what determines whether GRHL3 binds to its PWM in vivo, we analyzed GRHL3 bound sites (ChIP peaks containing a GRHL3 PWM) versus genome-wide GRHL3 PWM sites for different genomic features, including DNA methylation status in primary epidermal keratinocytes (60), evolutionary sequence conservation, $\mathrm{CpG}$ islands, repetitive sequences, and GC content (Supplemental Table 3 and ref. 61). Using 100 random shuffles of our original peak files from the 3 treatments and the peaks shared across all treatments (controlling for peak size and chromosomal distribution), we found that both evolutionary sequence conservation and presence of epidermal DNA methylation showed highly significant enrichment in the bound versus non-bound GRHL3 PWM sites and that the highest-confidence peaks common among the different experiments showed even greater enrichment
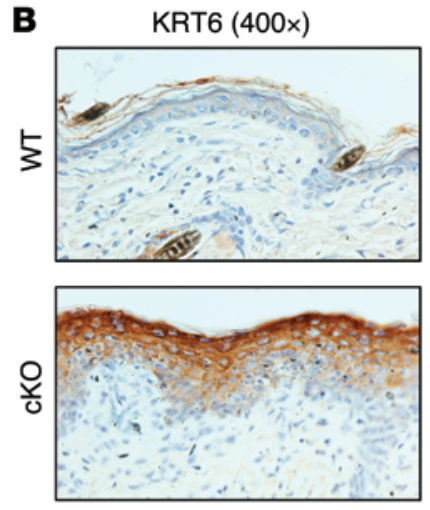

Recovery day 12
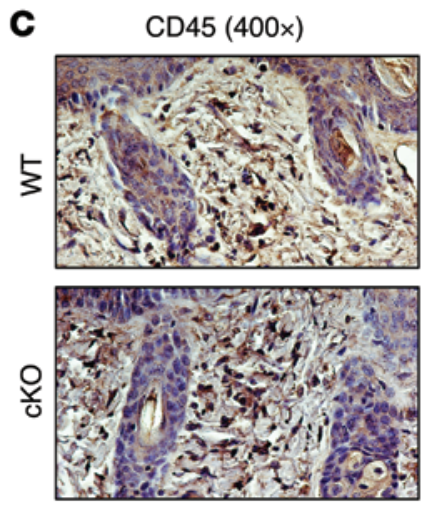

Recovery day 4

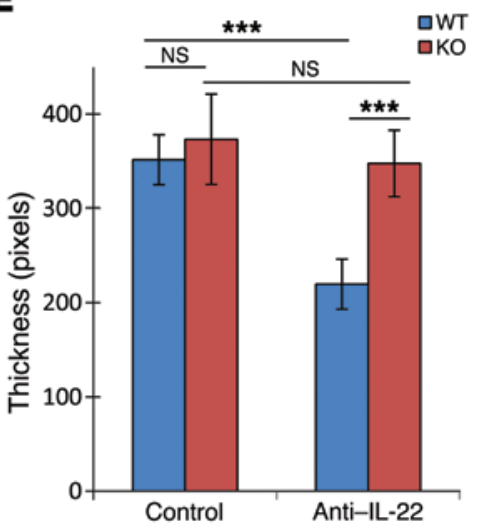

Figure 5. Grhl3 is required for healing of immune-mediated epidermal lesions. (A) H\&E of WT and Grhl3 cKO skin before treatment and at day 4 , recovery day 4 , and recovery day 12 of $5 \%$ IMQ treatment. Original magnification, $\times 200$. (B) KRT6 IHC of WT and Grh/3 cKO skin at recovery day 12 of $5 \%$ IMQ treatment. Original magnification, $\times 400$. (C) CD45 $\mathrm{IHC}$ staining of dermal immune cell infiltration at recovery day 4 of 5\% IMQ treatment. Original magnification, $\times 400$. (D) $H \& E$ of WT and Grhl3 cKO skin at day 3 of $5 \%$ IMQ treatment, with either control or anti-IL-22 treatment prior to IMQ application. Original magnification, $\times 200$. (E) Epidermal thickness at day 3 of $5 \%$ IMQ treatment with either control or anti-IL-22 treatment prior to IMQ application ( $n=4$ [WT and Grh/3 cKO]). ${ }^{* * *} P<0.001$; NS, $P>0.05$.

of these features (Figure 6G and Supplemental Table 3). Together, these data suggest that (a) under conditions of IMQ treatment, GRHL3 associates with many genomic sites that have not been identified as high-affinity binding sites in in vitro experiments; (b) although previously identified GRHL3 binding sites are statistically enriched in GRHL3 peaks, GRHL3 associates with many locations in the genome lacking these features; (c) many high-affinity GRHL3 binding sites remain unoccupied in the genome; (d) GRHL3 may associate with chromatin via recruitment by other transcription factors; and (e) GRHL3 preferentially associates with those sites found in regions marked by sequence conservation and epidermal DNA methylation, indicative of gene-regulatory regions.

GRHL3 regulates distinct gene batteries in embryonic epidermal differentiation and adult epidermal barrier reformation after injury. 

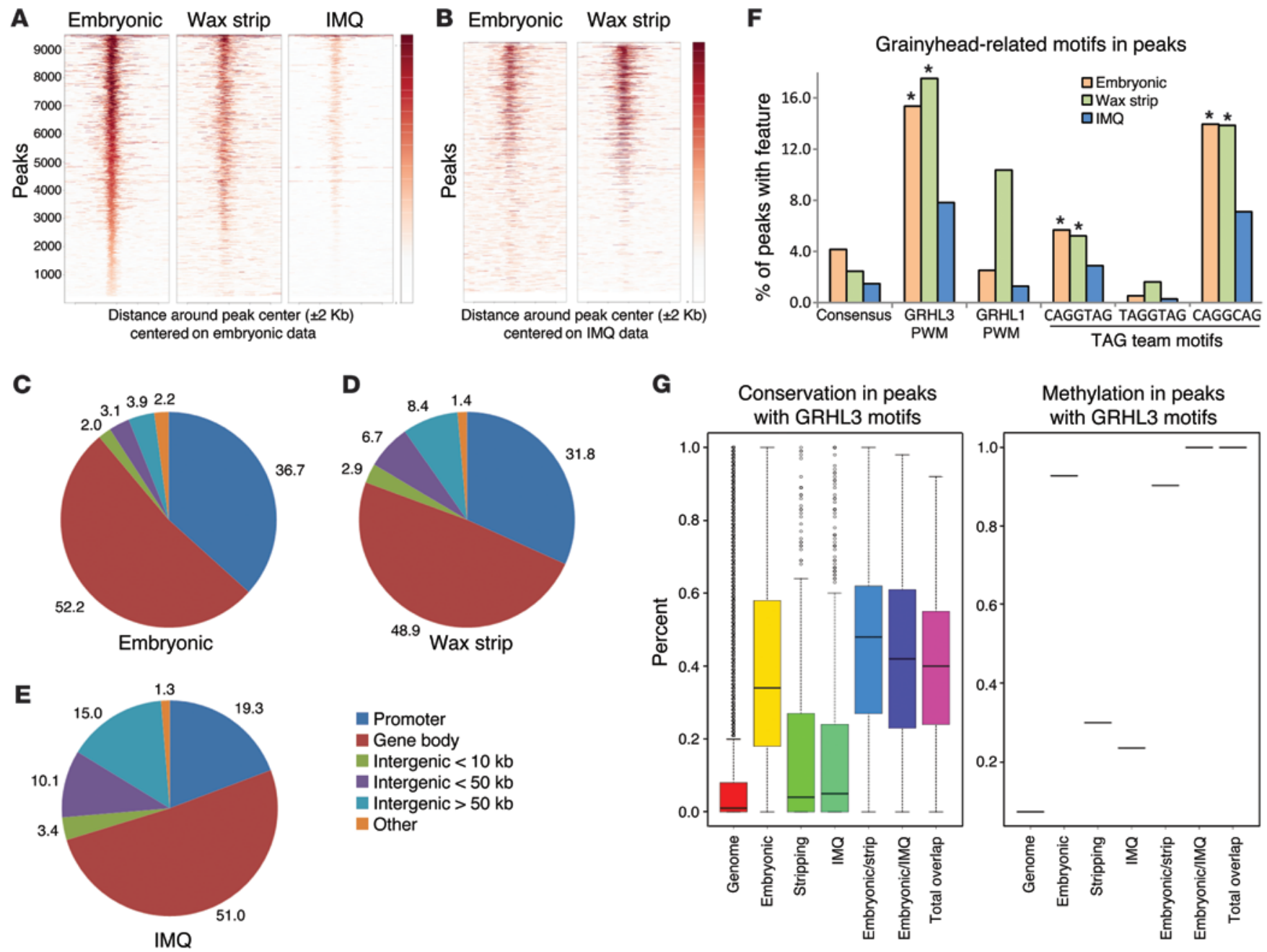

Figure 6. GRHL3 genomic binding is condition dependent. (A) Heatmaps showing the similarity among GRHL3 ChIP-Seq peaks in all 3 experiments centered on a single embryonic replicate. Left: Similarity between 2 independent replicates of E16.5 skin. Middle and right: Similarity between GRHL3 binding at E16.5 compared with wax-stripped (singlicate) and IMQ-treated (singlicate) epidermis. (B) Similarity among GRHL3 ChIP-Seq peaks in all 3 experiments centered on IMQ peaks. (C-E) Genomic distribution of GRHL3 binding at E16.5 (C, $n=2)$, after wax stripping (D, $n=1)$, and on IMQ treatment day $4(\mathbf{E}, n=1)$. (F) Fraction of CRHL3 ChIP-Seq peaks with known in vitro grainyhead motifs. ${ }^{*} P<0.05$, all 3 conditions. (G) Percent overlap of genomic features (left, evolutionary conservation; right, DNA methylation) with ChIP peaks containing GRHL3 PWM motifs compared with GRHL3 PWM motifs genome-wide (Genome). Boxes denote IQR, lines denote median, upper whiskers denote quartile $3+1.5 \times I Q R$, lower whiskers denote quartile $1-1.5 \times I Q R$, outliers denote values exceeding quartile $3+1.5 \times 1 \mathrm{Q}$.

Of the 2,471 differentially regulated genes in Grhl3 KO skin from E14.5 through E18.5 (Supplemental Figure 9, A and B, and Supplemental Table 4), 796 could be linked to a nearby embryonic GRHL3 ChIP peak ( $\pm 50 \mathrm{~kb}$ from the TSS; overlap significant, $P \leq 1.58 \times 10^{-10}$; Figure 7A and Supplemental Figure 9B). Among these were the epidermal differentiation- and epidermal barrierassociated genes Evpl, Lor, Tgm1, Elovl1, Elovl7, Smpd1, and Cldn6 (Supplemental Figure 12A); the gene ontology showed enrichment in lipid metabolism, GTPase signaling, epithelial differentiation, and cellular ion homeostasis (Figure 7B and Supplemental Figure 9, C-E). Thus, during epidermal development, GRHL3 appears to directly regulate the expression of a number of key genes required for normal epidermal differentiation and barrier formation.

During adult wax stripping recovery, there were 1,243 differentially regulated genes in Grhl3 cKO epidermis (Figure 7C, Supplemental Figure 10A, and Supplemental Table 5); these genes showed enrichment in lipid metabolism, epithelial differentiation, and ECM structure and organization (Supplemental Figure 9B). Of these genes, 326 could be linked to a nearby wax stripping ChIP peak (overlap significant, $P \leq 0.0381 ; \pm 50 \mathrm{~kb}$ ); the direct targets showed enrichment in morphogenesis, differentiation, and epithelium development (Figure 7D). Many gene targets in wax-stripped skin were involved in epidermal differentiation and barrier formation processes, including members of the Sprr family, lipid processing genes (Elovl3, Elovl4, and Alox8), cell death-related genes (Bnip3, Scrib, and Trp53bp2), and cell adhesion genes (Ctnnal1, Pvrl4, Cyr61, Itga3, Lama1, and Lamc1) (Supplemental Figure 12B). These data showed that GRHL3 also targets and regulates epidermal barrier-related genes after physical barrier disruption, although to a large extent, the specific targets were different than those in embryonic development (Supplemental Figure 12E). 


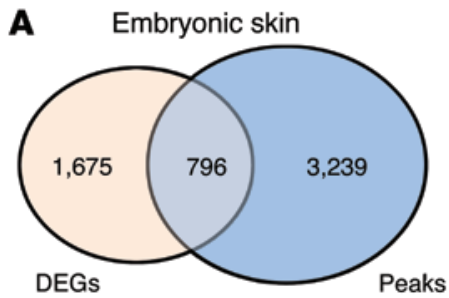

C Adult skin wax stripping
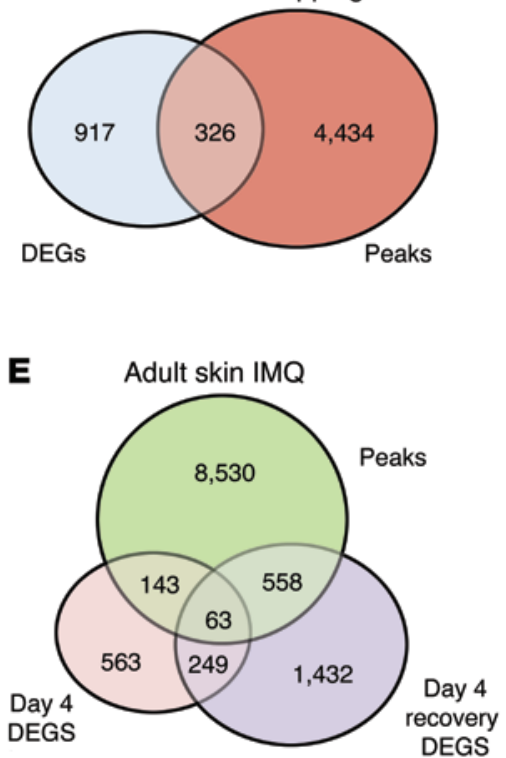

B

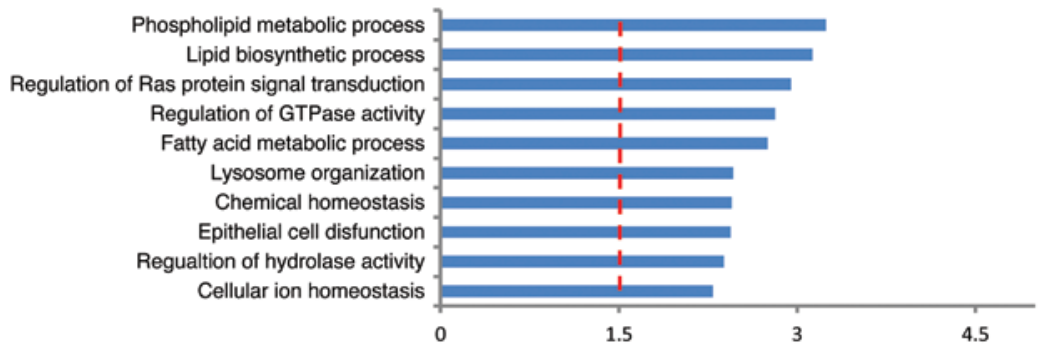

D

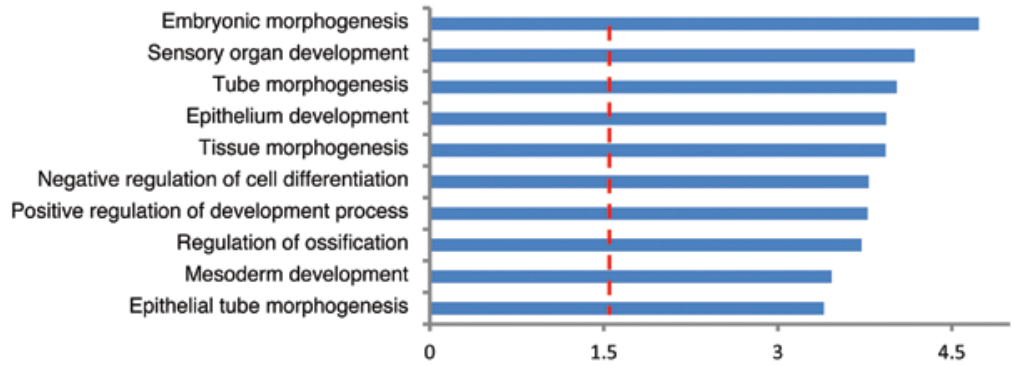

$\mathbf{F}$

Positive regulation of developmental process Defense response Cyclic-nucleotide-mediated signaling Regulation of cell division Inflammatory response Positive regulation of cell differentiation Negative regulation of cell differentiation Regulation of neurogenesis Positive regulation of transcription from RNA Pol-II prom Positive regulation of biosynthetic process

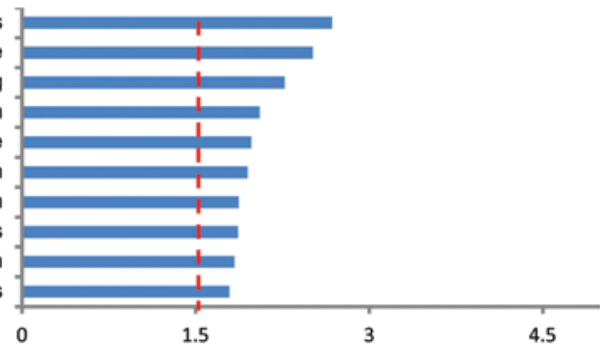

G

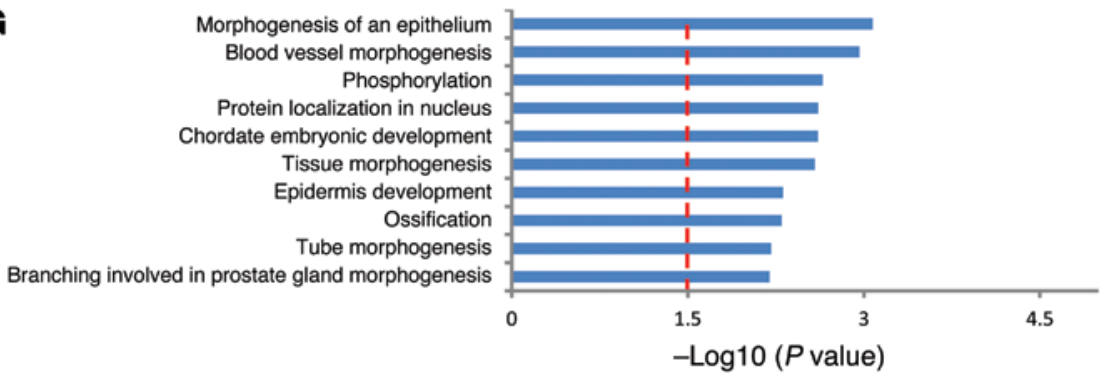

Figure 7. GRHL3 genomic binding, target regulation, and gene ontology. (A) Overlap between E16.5 GRHL3 ChIP peaks and DEGs (Grh/3 deletion in embryonic skin). (B) Gene ontology of GRHL3 embryonic targets (embryonic DEGs with proximal embryonic GRHL3 peaks; within +10 to $-5 \mathrm{~kb}$ of their TSS). (C) Overlap between wax-stripped GRHL3 ChIP peaks and DEGs (Grh/3 CKO wax-stripped epidermis). (D) Gene ontology of GRHL3 wax stripping targets (wax stripping DEGs with proximal wax stripping GRHL3 peaks). (E) Overlap between IMQ GRHL3 ChIP peaks and DEGs (Grh/3 cKO IMQ epidermis). (F and G) Gene ontology of GRHL3 IMQ targets (IMQ DEGs with proximal IMQ GRHL3 peaks) at day 4 of treatment (F) and day 4 of recovery (G).

GRHL3 regulates genes involved in the suppression of inflammation during immune-mediated epidermal damage. To understand the role of Grhl3 in immune-mediated epidermal injury, we compared gene expression in WT and Grhl3 cKO epidermis at day 4 of $5 \%$ IMQ treatment and at day 4 of recovery from 5\% IMQ treatment, identifying 769 and 2,053 differentially regulated genes, respectively (Figure 7E, Supplemental Figure 11, A and B, and Supplemental Tables 6 and 7). Enriched gene ontology of DEGs during IMQ treatment included retinoid metabolism, blood vessel development, lipid localization, and epithelium development, while different categories became more prominent during recovery from IMQ treatment, including immune response, epidermis development, and epidermal cell differentiation (Supplemental Figure 11, $\mathrm{C}$ and D). Most strikingly, during recovery from IMQ treatment, there was strong overexpression of numerous epidermal injury response- and psoriatic lesion-associated genes, including the alarmin S100a8, the defensin Def $3 b$, and Tslp, a known important immune-modulatory gene in skin disease; these genes were not affected by Grhl3 deletion during embryogenesis.

Nearby GRHL3 binding in the IMQ ChIP-Seq data ( $\pm 50 \mathrm{~kb})$ was found in 212 (overlap NS, $P \leq 0.897$ ) and 543 (overlap significant, $P \leq 0.0375$ ) of the differentially regulated genes in Grhl3 cKO skin at IMQ treatment day 4 and recovery day 4 , respectively (Figure $7 \mathrm{E})$. The direct targets at day 4 of treatment showed enrichment in defense response, inflammatory response, regulation of cell differentiation, and regulation of transcription (Figure 7F), whereas during recovery, epithelium morphogenesis and epidermis development became more prominent (Figure 7G). Direct tar- 
gets of GRHL3 in IMQ-treated epidermis included several important epidermal-related genes, such as transcription factors (Ovol1 and Gata6), lipid metabolism genes (Alox12e, Elovl3, and Smpd1), cell death-related genes (Shisa5, Rhob, and Jak2), and cell adhesion molecules (Dsg1b and Cdh24) (Supplemental Figure 12, C and D). Especially evident in the IMQ model were immune-related damage response targets (Il1a, Pparg, Tlr3, Defb14, Defb7, Defb3, and Stab1), cAMP-mediated signaling (Pthlh, S1pr3, and Drd1a), and retinoid metabolism (Rdh9, Lrat, and Rdh1). We concluded that although it regulates epidermal barrier formation under all 3 conditions (embryonic development, wax stripping, and IMQ treatment), GRHL3 plays a more prominent role in regulating injury- and inflammatory-related pathways during the repair of immune-mediated epidermal damage. In general, the differentially expressed genes (DEGs) in response to Grhl3 deletion were specific to each of the 3 conditions (Supplemental Figure 12E). Furthermore, GRHL3 tended to bind these genes only under the condition in which their expression was altered by Grhl3 deletion. Only 71 and 104 (wax strip and IMQ, respectively) GRHL3 target genes overlapped with targets during embryogenesis, with just 25 common to all 3 conditions, including the key epidermal genes Lor, Ppl, and Smpd1 (Supplemental Figure 12, A-E). These results indicate that chromatin binding of GRHL3 is highly dependent on the functional state of the epidermis, such as after different types of epidermal injury.

\section{Discussion}

Our present findings demonstrate the importance of a GRHL3controlled repair pathway in skin lesions caused by immune-mediated epidermal barrier disruption. Several key findings support this conclusion. First, although GRHL3 was expressed at a low level in the adult mouse epidermis, and appeared to be dispensable for normal epidermal homeostasis, the gene was upregulated upon both mechanical and immune-mediated epidermal damage. Second, in Grhl3 deleted mice, epidermal hyperplasia caused by mechanical, chemical, and immunological barrier disruption was easier to induce and failed to resolve properly. Third, in human psoriasis, GRHL3 was consistently upregulated in lesions, where its expression correlated with elevation of IL-17 activity. Fourth, GRHL3 expression was reduced after psoriatic lesions were resolved by treatment with anti-TNF, anti-IL-23, or anti-IL-17R. Fifth, GRHL3 bound to several barrier gene targets in human psoriatic epidermal lesions. By analogy to tumor suppressor pathways, we surmise that the GRHL3-controlled repair pathway acts to suppress epidermal hyperplasia in diseases such as psoriasis (Supplemental Figure 13).

Our ChIP-Seq and gene expression data indicated that GRHL3 regulates barrier formation during epidermal development through direct regulation of a number of genes encoding factors required for epidermal differentiation and barrier formation. Interestingly, the renewed requirement for GRHL3 in adult barrier repair was not simply a reactivation of the embryonic gene expression program. Rather, GRHL3 targeted distinct groups of genes after adult physical barrier injury and immune-mediated epidermal injury. Some of the GRHL3 targets observed in IMQ-mediated epidermal damage are involved in lipid processing, cell death, and cell adhesion, all known to be important for epidermal differentiation, thus accounting for its repair-promoting effect in immune-mediated skin disease. However, after injury in the adult epidermis, GRHL3 also suppressed epidermally expressed genes encoding proinflammatory factors (including Il1a, Stat1, Tlr3, Tnfrsf18, and Irf6). Consistent with this result, we found that during low-dose IMQ treatment, there was enhanced immune cell infiltration in Grhl3 cKO skin. Thus, our data suggest that GRHL3 suppresses epidermal hyperplasia in part by restraining immune activation mechanisms in the epidermis in response to perturbation.

Data on GRHL3 targets in human keratinocytes (35), combined with previous human psoriasis datasets (31-33), suggest that not all of the normal differentiation-related targets downstream of GRHL3 respond to the GRHL3 upregulation in psoriasis lesions. One explanation for this intriguing finding is that the psoriatic environment counteracts epidermal barrier repair activities downstream of GRHL3, possibly through alterations in GRHL3 binding partners, disease-related epigenetic alterations, or posttranslational modifications to GRHL3 itself. These genes may also become targeted by inflammatory-related DNA-binding proteins that counteract GRHL3's actions.

There is strong evidence that secondary to immune pathogenetic mechanisms, epidermal factors contribute to the psoriasis disease phenotype through the release of cytokines and chemokines $(7,21,62-65)$. While a primary role for the epidermis has been clearly shown in atopic dermatitis (66), a primary role for the epidermis remains open to debate in the psoriasis field. However, GWAS studies identifying associations with epidermally expressed targets - including the late cornified envelope locus (67), connexin $26(68,69)$, and $\operatorname{KLF} 4(43,70)$, a GRHL3 target - support such mechanisms. While these previous studies implicate a defective barrier in the disease, our present work is suggestive of an active epidermal repair pathway as a diseasesuppressing activity. Genetic studies in psoriasis have thus far focused on the association between genetic variants and disease occurrence, not on disease severity or lesional healing rates. As our data point to a primary role for GRHL3 in repair of lesions, genetic changes affecting GRHL3 expression or function are more likely to correlate strongly with disease activity and treatment resistance. Consistent with this notion, we found that loss of Grhl3 conferred resistance to anti-IL-22 therapy, which normally counteracts IMQ-induced epidermal hyperplasia. A more thorough understanding of epidermal repair pathways in psoriasis may lead to discoveries of treatment targets that could both counteract lesional severity and enhance their resolution.

\section{Methods}

Generation of Grhl3 cKO mice and use of Grhl3-Cre/LacZ reporter mice. Grhl3 floxed mice (C57BL/6J), generated previously by the Andersen lab (16), were crossed with Krt14-Cre mice (gift of S. Millar, University of Pennsylvania School of Medicine, Philadelphia, Pennsylvania, USA; ref. 25) to produce both heterozygous-flox and homozygous-flox Crepositive mice (Krt14-Cre Grhl3 $3^{\mathrm{fl}-}$ and $\mathrm{Krt14}$-Cre Grhl3 $3^{\mathrm{A} / \mathrm{f}}$, respectively). Grhl3-Cre/LacZ reporter mice (26) were purchased from MMRRC.

Immunohistochemistry (IHC). Mouse back skin was fixed in 10\% formalin and embedded in paraffin. Sectioning and staining was performed as previously described (71). $\mathrm{BrdU}(10 \mathrm{mg} / \mathrm{ml})$ was injected i.p. 4 hours prior to sacrificing the mice, and slides were treated with $1 \mathrm{~N} \mathrm{HCl}$ 
for 1 hour at $37^{\circ} \mathrm{C}$, followed by $0.1 \mathrm{M}$ Borate to neutralize the acid before addition of the BrdU antibody (Roche, 11170376001). Other antibodies used were as follows: K10 (Covance, PRB-159P), K6 (Covance, PRB169P), CD45 (R\&D Systems, MAB114), LacZ (Abcam, ab9361).

Bromophenol blue staining and TEM. Skin was fixed in 2\% paraformaldehyde prior to sectioning and bromophenol blue staining or TEM. For TEM, ultrathin sections were visualized in a FEI/Phillips CM-20 electron microscope.

Wax stripping, IMQ application, and IL-22 injection. Mice were anesthetized using ketamine $(100 \mathrm{mg} / \mathrm{kg})$ and xylazine $(10 \mathrm{mg} / \mathrm{kg})$ prior to shaving of the backskin using electric clippers. Wax strips covering the shaved area were applied and then stripped off (3 strips per mouse). Undiluted 5\% IMQ (Aldara) or IMQ diluted with over-thecounter skin cream (final concentrations, $1 \%$ and $0.25 \%$ ) was applied daily for 3-6 days $(0.625 \mathrm{mg} / \mathrm{mouse} / \mathrm{d})$. Anti-IL-22 antibody was injected $(500 \mu \mathrm{g})$ i.p. 1 day prior to IMQ application.

RNA extraction. Tissue was collected, and the epidermal portion was separated from the dermal portion using a 1:3 dilution of dispase (Stem Cell Technologies, catalog no. 07913 ) at $37^{\circ} \mathrm{C}$ for 1 hour. The epidermis was then lysed in TRIzOL, followed by chloroform extraction and RNA purification from the aqueous phase using Ambion PureLink RNA mini kit per the manufacturer's protocol. RNA concentration and quality were quantified on a NanoDrop.

Microarray analysis. All experiments were performed with biological duplicates as previously described (35), except Affymetrix Mouse Gene 1.0 ST arrays were used and washed according to the manufacturer's recommendations (Affymetrix). DEGs were analyzed with Cyber-T (72) and considered significant for $P$ values less than 0.01 (for analysis of publicly available human psoriasis, a fold cutoff of \pm 1.5 was applied). Gene Ontology analysis was performed on all datasets using DAVID. The microarray data discussed herein were deposited in GEO (accession no. GSE59384).

Quantitative real-time PCR. For mRNA expression analysis, cDNA was prepared using iScript cDNA kit (Biorad Laboratories), and RTPCR was performed using SsoFast EvaGreen (Biorad Laboratories) master mixes on a CFX384 Real-Time PCR Detection System (Biorad Laboratories). $\beta$-Actin was used as the endogenous control (see Supplemental Table 8 for primer sequences).

ChIP assays. ChIP assays with GRHL3 antibodies were performed and analyzed as previously described (35). Epidermis was collected for ChIP in the same manner as for RNA isolation; enrichment was calculated above an IgG (Sigma-Aldrich, catalog no. 15006-10MG) control IP and normalized to a negative control genomic region (see Supplemental Table 8 for primer sequences).

ChIP-Seq. Sequencing libraries were generated for the GRHL3 and input samples as previously described (35) using NEB Next reagents and Illumina adaptors and oligonucleotides. 50-cycle single end sequencing was performed on an Illumina Hi-Seq 2000 Genome Analyzer, and the resulting reads were aligned using Bowtie (73), with only uniquely aligning reads retained. Peaks were called with MACS (74), and further analysis was performed using Galaxy. The ChIP-Seq data discussed herein were deposited in GEO (accession no. GSE52648).

Statistics. For all bar graphs, data are presented as mean \pm SEM. For all box and whisker plots, the bounds of the boxes represent interquartile range (IQR), lines within the boxes represent median, and whiskers denote maximum and minimum values (unless otherwise indicated). For gene expression overlaps, significance was assessed using a hyper- geometric $P$ value calculation. For ChIP-Seq overlaps, significance was assessed using HOMER (56), which performs a hypergeometric distribution on the bases covered by peaks. To test the significance of overlap between the ChIP-Seq and DEGs from the microarrays, we took 100 random shuffles of the ChIP-Seq peak files, associated each peak with its nearest gene, and assessed the significance of DEG overlap by estimating the parameters of normal distribution and calculating a $z$ score of the observed DEG overlap, which was tested for significance at $\alpha<0.05$. This test was additionally performed on the overlap of the common ChIP-Seq peaks and the common DEGs at $\alpha<0.05$. Similarly, to test for significance of motif occurrence, PWMs for GRHL3 were searched across all peaks using HOMER and MotifMap (59) in the ChIP-Seq peaks as well as for 100 random shuffles of peaks, and significance was assessed using $z$ score at $\alpha<0.05$. We assessed numerous peak level features for significance - distance to nearest TSS, percent CpG, percent GC, conservation, CpG methylation overlap, and RepeatMask overlap - for peaks containing a GRHL3 PWM site versus genome-wide sites with GRHL3 PWM. Using a logodd score cutoff tuned to a known GRHL3 site, we used MotifMap to first identify all locations of the GRHL3 PWM in the genome (mm9) above the cutoff, expanded to $300 \mathrm{bp}$ (roughly the average peak length for the ChIP-Seq peaks). Similarly, ChIP-Seq peaks were filtered for those containing at least 1 GRHL3 site above the cutoff. For each peak level feature, we tested for a significance difference in the ChIP-Seq peaks containing GRHL3 compared with genome background using a 1-sided Wilcoxon rank-sum test for the numeric features (distance, percent CpG, percent GC, and conservation) and Fisher's exact test on the $\mathrm{CpG}$ methylation and RepeatMasker overlap counts.

To test the significance of the overlapping genes between GRHL3 siRNA and human psoriasis with the GRHL3 ChIP-Seq peaks, 10,000 simulation trials were performed. In each trial, 3 gene sets were chosen randomly consisting of the matching total numbers for each respective dataset from among the genes found to be expressed in both keratinocytes and human psoriasis. Of the 10,000 trials performed, only 485 yielded a 3-way overlap greater than 45 genes; consequently, the simulated $P$ value was 0.0485 (i.e., 485/10,000).

Cytokine signatures for psoriasis patients were calculated using a previously described method (34). In brief, signature scores were calculated using the 250 genes most strongly induced by a given cytokine treatment in cultured keratinocytes (or reconstituted epidermis). To identify these 250 genes, we first identified the 375 genes most strongly induced by the cytokine treatment based on $P$ values obtained by testing for differential expression (cytokine-treated cells versus controls; empirical Bayes linear models as implemented in the limma package for R Bioconductor). These 375 genes were then sorted according to fold change estimates, and we selected the 250 genes with the highest fold change estimate (treated cells/controls). For each patient, cytokine signature scores were then calculated as the weighted arithmetic mean of $\mathrm{PP} / \mathrm{PN}$ fold changes (i.e., psoriasis lesion/uninvolved skin) among these 250 genes, with greater weight assigned to those genes most strongly induced in cytokine-treated keratinocytes. Finally, for each patient, we calculated the PP/PN fold change estimate for GRHL3 and determined whether these fold change estimates correlated with cytokine signature scores among all patients included in our meta-cohort ( $n=163$ patients). To ensure that signature scores were not themselves based on GRHL3 expression, in all cases we excluded GRHL 3 from the set of 250 genes upon which cytokine signature scores were based. 
Study approval. All mouse experiments were approved by the UCI IACUC (approval no. 2001-2239). Human skin biopsy collection was approved by the University of Michigan Medical School IRB (approval no. HUM00019384), and subjects provided informed consent prior to their participation in the study.

\section{Acknowledgments}

This work was supported by NIH grant AR44882 and the Irving Weinstein Foundation (to B. Andersen); by NIH fellowship T32HD60555 (to W.M. Gordon); by NIH Fellowship 1F32AR06535601A1 (to H. Ho); by the National Psoriasis Foundation, the A. Alfred Taubman Medical Research Institute (via the Frances and Kenneth Eisenberg Emerging Scholar Award), the Doris Duke Foundation, and NIH grant K08AR060802 (to J.E. Gudjonsson); by an American Skin Association Carson Family Research Scholar Award in
Psoriasis (to W.R. Swindell); and by NIH grants LM010235 and NLM T15 LM07443 and NSF grant IIS-0513376 (to P.F. Baldi). We also acknowledge the support of the UCI Institute for Clinical and Translational Science (UL1 TR000153), the UCI Institute for Genomics and Bioinformatics, a hardware donation by NVIDIA, Yuzo Kanomata for additional support of our computational infrastructure, Alice Liang (NYU Medical College) for TEM specimen preparation, Jian-Guo Zheng (Laboratory for Electron and X-ray Instrumentation [LEXI], UCI) for TEM, Sarah Millar for the gift of Krt14-Cre mice, and the UCI High-Throughput Genome Facility for processing microarrays and ChIP-Seq libraries.

Address correspondence to: Bogi Andersen, University of California, Irvine Sprague Hall, Room 206, Irvine, California 92697, USA. Phone: 949.824.9093; E-mail: bogi@uci.edu.
1. Cai Y, Fleming C, Yan J. New insights of T cells in the pathogenesis of psoriasis. Cell Mol Immunol. 2012;9(4):302-309.

2. Ghoreschi K, Weigert C, Röcken M. Immunopathogenesis and role of T cells in psoriasis. Clin Dermatol. 2007;25(6):574-580.

3. Meephansan J, Komine M, Tsuda H, Karakawa M, Tominaga S, Ohtsuki M. Expression of IL-33 in the epidermis: The mechanism of induction by IL-17. J Dermatol Sci. 2013;71(2):107-114.

4. Bouchaud G, et al. Epidermal IL-15R $\alpha$ acts as an endogenous antagonist of psoriasiform inflammation in mouse and man. J Exp Med. 2013;210(10):2105-2117.

5. Kitoh A, Nomura T, Kabashima K. TGF $\beta 1$, an epidermal controller of skin dendritic cell homeostasis. J Invest Dermatol. 2013;133(1):9-11.

6. Sarra M, et al. IL-21 promotes skin recruitment of CD4(+) cells and drives IFN- $\gamma$ dependent epidermal hyperplasia. J Immunol. 2011;186(9):5435-5442.

7. Muhr P, Zeitvogel J, Heitland I, Werfel T, Wittmann M. Expression of interleukin (IL)-1 family members upon stimulation with IL-17 differs in keratinocytes derived from patients with psoriasis and healthy donors. Br J Dermatol. 2011;165(1):189-193.

8. Buchau AS, Gallo RL. Innate immunity and antimicrobial defense systems in psoriasis. Clin Dermatol. 2007;25(6):616-624.

9. Albanesi C, De Pita O, Girolomoni G. Resident skin cells in psoriasis: a special look at the pathogenetic functions of keratinocytes. Clin Dermatol. 2007;25(6):581-588.

10. Mee JB, Cork MJ, di Giovine FS, Duff GW, Groves RW. Interleukin-1: a key inflammatory mediator in psoriasis? Cytokine. 2006;33(2):72-78.

11. Chang EY, et al. T-cell activation is potentiated by cytokines released by lesional psoriatic, but not normal, epidermis. Arch Dermatol. 1992;128(11):1479-1485.

12. Bergboer JG, Zeeuwen PL, Schalkwijk J. Genetics of psoriasis: evidence for epistatic interaction between skin barrier abnormalities and immune deviation. J Invest Dermatol. 2012;132(10):2320-2321.

13. Stramer B, Martin P. Cell biology: master regulators of sealing and healing. Curr Biol.
2005;15(11):R425-R427.

14. Darido C, et al. Targeting of the tumor suppressor GRHL3 by a miR-21-dependent proto-oncogenic network results in PTEN loss and tumorigenesis. Cancer Cell. 2011;20(5):635-648.

15. Caddy J, et al. Epidermal wound repair is regulated by the planar cell polarity signaling pathway. Dev Cell. 2010;19(1):138-147.

16. Yu Z, et al. The Grainyhead-like epithelial transactivator Get-1/Grhl3 regulates epidermal terminal differentiation and interacts functionally with LMO4. Dev Biol. 2006;299(1):122-136.

17. Bhandari A, et al. The Grainyhead transcription factor Grhl3/Get1 suppresses miR-21 expression and tumorigenesis in skin: modulation of the miR-21 target MSH2 by RNA-binding protein DND1. Oncogene. 2013;32(12):1497-1507.

18. Kudryavtseva EI, et al. Identification and characterization of Grainyhead-like epithelial transactivator (GET-1), a novel mammalian Grainyheadlike factor. Dev Dyn. 2003;226(4):604-617.

19. Ting SB, et al. A homolog of Drosophila grainy head is essential for epidermal integrity in mice. Science. 2005;308(5720):411-413.

20. Peyrard-Janvid M, et al. Dominant mutations in GRHL3 cause Van der Woude Syndrome and disrupt oral periderm development. Am J Hum Genet. 2014;94(1):23-32.

21. Swindell WR, Johnston A, Xing X, Voorhees JJ, Elder JT, Gudjonsson JE. Modulation of epidermal transcription circuits in psoriasis: new links between inflammation and hyperproliferation. PLoS One. 2013;8(11):e79253.

22. van der Fits L, et al. Imiquimod-induced psoriasis-like skin inflammation in mice is mediated via the IL-23/IL-17 axis. J Immunol. 2009;182(9):5836-5845.

23. Swindell WR, et al. Genome-wide expression profiling of five mouse models identifies similarities and differences with human psoriasis. PLoS One. 2011;6(4):e18266.

24. Van Belle AB, et al. IL-22 is required for imiquimod-induced psoriasiform skin inflammation in mice. J Immunol. 2012;188(1):462-469.

25. Andl T, et al. Epithelial Bmprla regulates differentiation and proliferation in postnatal hair follicles and is essential for tooth development. Development. 2004;131(10):2257-2268.
26. Camerer E, et al. Local protease signaling contributes to neural tube closure in the mouse embryo. Dev Cell. 2010;18(1):25-38.

27. Tsai JC, Shen LC, Sheu HM, Lu CC. Tape stripping and sodium dodecyl sulfate treatment increase the molecular weight cutoff of polyethylene glycol penetration across murine skin. Arch Dermatol Res. 2003;295(4):169-174.

28. Barrett T, et al. NCBI GEO: archive for functional genomics data sets - update. Nucleic Acids Res. 2013;41(Database issue):D991-D995.

29. Wolf R, Orion E, Ruocco E, Ruocco V. Abnormal epidermal barrier in the pathogenesis of psoriasis. Clin Dermatol. 2012;30(3):323-328.

30. Ghadially R, Reed JT, Elias PM. Stratum corneum structure and function correlates with phenotype in psoriasis. JInvest Dermatol. 1996;107(4):558-564.

31. Yao Y, et al. Type I interferon: potential therapeutic target for psoriasis? PLoS One. 2008;3(7):e2737.

32. Suárez-Fariñas M, Li K, Fuentes-Duculan J, Hayden K, Brodmerkel C. Expanding the psoriasis disease profile: interrogation of the skin and serum of patients with moderate-to-severe psoriasis. J Invest Dermatol. 2012;132(11):2552-2564.

33. Nair RP, et al. Genome-wide scan reveals association of psoriasis with IL-23 and NF- $\kappa$ B pathways. Nat Genet. 2009;41(2):199-204.

34. Swindell WR, Johnston A, Voorhees JJ, Elder JT, Gudjonsson JE. Dissecting the psoriasis transcriptome: inflammatory- and cytokine-driven gene expression in lesions from 163 patients. BMC Genomics. 2013;14:527.

35. Hopkin AS, et al. GRHL3/GET1 and trithorax group members collaborate to activate the epidermal progenitor differentiation program. PLoS Genet. 2012;8(7):e1002829.

36. Lobito AA, et al. Murine insulin growth factor-like (IGFL) and human IGFL1 proteins are induced in inflammatory skin conditions and bind to a novel tumor necrosis factor receptor family member, IGFLR1. J Biol Chem. 2011;286(21):18969-18981.

37. Pennisi DJ, et al. Crim1KST264/KST264 mice display a disruption of the Crim 1 gene resulting in perinatal lethality with defects in multiple organ systems. Dev Dyn. 2007;236(2):502-511.

38. Kurtz A, et al. Differential regulation of a fibro- 
blast growth factor-binding protein during skin carcinogenesis and wound healing. Neoplasia. 2004;6(5):595-602.

39. Kurtz A, Wang HL, Darwiche N, Harris V, Wellstein A. Expression of a binding protein for FGF is associated with epithelial development and skin carcinogenesis. Oncogene. 1997;14(22):2671-2681.

40. Rojek AM, et al. Defective glycerol metabolism in aquaporin 9 (AQP9) knockout mice. Proc Natl Acad Sci U S A. 2007;104(9):3609-3614.

41. Grether-Beck S, et al. Urea uptake enhances barrier function and antimicrobial defense in humans by regulating epidermal gene expression. J Invest Dermatol. 2012;132(6):1561-1572.

42. Hwang J, Kita R, Kwon HS, Choi EH, Lee SH, Udey MC. Epidermal ablation of Dlx 3 is linked to IL-17-associated skin inflammation. Proc Natl Acad Sci US A. 2011;108(28):11566-11571.

43. Tsoi LC, et al. Identification of 15 new psoriasis susceptibility loci highlights the role of innate immunity. Nat Genet. 2012;44(12):1341-1348.

44. Yang JM, Sim SM, Kim HY, Park GT. Expression of the homeobox gene, HOPX, is modulated by cell differentiation in human keratinocytes and is involved in the expression of differentiation markers. Eur J Cell Biol. 2010;89(7):537-546.

45. Haveri $\mathrm{H}$, et al. Transcription factors GATA-4 and GATA-6 in normal and neoplastic human gastrointestinal mucosa. BMC Gastroenterol. 2008;8:9.

46. Belaguli NS, Zhang M, Rigi M, Aftab M, Berger DH. Cooperation between GATA4 and TGF- $\beta$ signaling regulates intestinal epithelial gene expression. Am J Physiol Gastrointest Liver Physiol. 2007;292(6):G1520-G1533.

47. Guillemot L, Spadaro D, Citi S. The junctional proteins cingulin and paracingulin modulate the expression of tight junction protein genes through GATA-4. PLoS One. 2013;8(2):e55873.

48. Bigler J, Rand HA, Kerkof K, Timour M, Russell CB. Cross-study homogeneity of psoriasis gene expression in skin across a large expression range. PLoS One. 2013;8(1):e52242.

49. Russell CB, et al. Gene expression profiles normalized in psoriatic skin by treatment with brodalumab, a human anti-IL-17 receptor monoclonal antibody. J Immunol. 2014;192(8):3828-3836.
50. Sofen H, et al. Guselkumab (an IL-23-specific $\mathrm{mAb}$ ) demonstrates clinical and molecular response in patients with moderate-tosevere psoriasis. J Allergy Clin Immunol. 2014;133(4):1032-1040.

51. Swindell WR, et al. Cellular dissection of psoriasis for transcriptome analyses and the postGWAS era. BMC Med Genomics. 2014;7:27.

52. Lindroos J, et al. IL-23-mediated epidermal hyperplasia is dependent on IL-6. J Invest Dermatol. 2011;131(5):1110-1118.

53. Wu JK, Siller G, Strutton G. Psoriasis induced by topical imiquimod. Australas J Dermatol. 2004;45(1):47-50.

54. Fanti PA, Dika E, Vaccari S, Miscial C, Varotti C. Generalized psoriasis induced by topical treatment of actinic keratosis with imiquimod. Int $J$ Dermatol. 2006;45(12):1464-1465.

55. Rajan N, Langtry JA. Generalized exacerbation of psoriasis associated with imiquimod cream treatment of superficial basal cell carcinomas. Clin Exp Dermatol. 2006;31(1):140-141.

56. Heinz S, et al. Simple combinations of lineagedetermining transcription factors prime cis-regulatory elements required for macrophage and $\mathrm{B}$ cell identities. Mol Cell. 2010;38(4):576-589.

57. Harrison MM, Botchan MR, Cline TW. Grainyhead and Zelda compete for binding to the promoters of the earliest-expressed Drosophila genes. Dev Biol. 2010;345(2):248-255.

58. Wilanowski T, et al. A highly conserved novel family of mammalian developmental transcription factors related to Drosophila grainyhead. Mech Dev. 2002;114(1):37-50.

59. Daily K, Patel VR, Rigor P, Xie X, Baldi P. MotifMap: integrative genome-wide maps of regulatory motif sites for model species. BMC Bioinformatics. 2011;12:495.

60. Bock C, et al. DNA methylation dynamics during in vivo differentiation of blood and skin stem cells. Mol Cell. 2012;47(4):633-647.

61. Karolchik D, et al. The UCSC Genome Browser database: 2014 update. Nucleic Acids Res. 2014;42(Database issue):D764-D770.

62. Johnston A, et al. Keratinocyte overexpression of IL-17C promotes psoriasiform skin inflamma- tion. J Immunol. 2013;190(5):2252-2262.

63. Lowes MA, Russell CB, Martin DA, Towne JE, Krueger JG. The IL-23/T17 pathogenic axis in psoriasis is amplified by keratinocyte responses. Trends Immunol. 2013;34(4):174-181.

64. Lee $\mathrm{Y}$, et al. S100A8 and S100A9 are messengers in the crosstalk between epidermis and dermis modulating a psoriatic milieu in human skin. Biochem Biophys Res Commun. 2012;423(4):647-653.

65. Wolf R, et al. Gene from a psoriasis susceptibility locus primes the skin for inflammation. Sci Transl Med. 2010;2(61):61ra90.

66. Palmer $\mathrm{CN}$, et al. Common loss-of-function variants of the epidermal barrier protein filaggrin are a major predisposing factor for atopic dermatitis. Nat Genet. 2006;38(4):441-446.

67. de Cid R, et al. Deletion of the late cornified envelope LCE3B and LCE3C genes as a susceptibility factor for psoriasis. Nat Genet. 2009;41(2):211-215.

68. Tang $\mathrm{H}$, et al. A large-scale screen for coding variants predisposing to psoriasis. Nat Genet. 2014;46(1):45-50.

69. Sun LD, et al. Association analyses identify six new psoriasis susceptibility loci in the Chinese population. Nat Genet. 2010;42(11):1005-1009.

70. Segre JA, Bauer C, Fuchs E. Klf4 is a transcription factor required for establishing the barrier function of the skin. Nat Genet. 1999;22(4):356-360.

71. Yu Z, Bhandari A, Mannik J, Pham T, Xu X, Andersen B. Grainyhead-like factor Get1/ Grhl3 regulates formation of the epidermal leading edge during eyelid closure. Dev Biol. 2008;319(1):56-67.

72. Kayala MA, Baldi P. Cyber-T web server: differential analysis of high-throughput data. Nucleic Acids Res. 2012;40(Web Server issue):W553-W559.

73. Langmead B, Trapnell C, Pop M, Salzberg SL. Ultrafast and memory-efficient alignment of short DNA sequences to the human genome. Genome Biol. 2009;10(3):R25.

74. Zhang Y, et al. Model-based analysis of ChIP-Seq (MACS). Genome Biol. 2008;9(9):R137.

75. Swindell WR, et al. Heterogeneity of inflammatory and cytokine networks in chronic plaque psoriasis. PLoS One. 2012;7(3):e34594. 